\title{
Existence of Time Periodic Solutions of New Classes of Nonlinear Problems
}

\author{
Luisa Toscano ${ }^{1}$, Speranza Toscano $^{2}$ \\ ${ }^{1}$ Dep. of Math. and Appl.’R. Caccioppoli”,Univ. of Naples “Federico II”, via Cintia, Monte S. Angelo, Italy \\ ${ }^{2}$ Dep. of Civil Ing,. Second Univ. of Naples, fac. of Ing., Aversa (CE), Italy
}

\section{Email address:}

luisa.toscano2@unina.it (L. Toscano), speranza.toscano@libero.it (S. Toscano)

\section{To cite this article:}

Luisa Toscano, Speranza Toscano. Existence of Time Periodic Solutions of New Classes of Nonlinear Problems. Pure and Applied Mathematics Journal. Vol. 4, No. 5, 2015, pp. 189-215. doi: 10.11648/j.pamj.20150405.11

\begin{abstract}
We study the existence of one or more weak periodic solutions of nonlinear evolution PDEs in a cylinder of $\mathrm{R}^{\mathrm{N}+1}$ with conditions on lateral surface by using the results connected to a general evolution variational equation depending on a parameter.
\end{abstract}

Keywords: Time Periodic, Evolution PDEs, Nonstationarity, Weak Periodic Solutions

\section{Introduction}

Let $0<T<\infty$. Let $\Omega \subseteq \mathrm{R}^{\mathrm{N}}$ be an open, bounded and connected set, with boundary $\partial \Omega \in C^{0,1}$.

For $\mathrm{N}=1$, the condition " $\partial \Omega \in C^{0,1}$ "means that $\Omega$ is a bounded open interval.

Let us set

$$
Q=\Omega \times] 0, T[\text { and } \Sigma=\partial \Omega \times] 0, T[
$$

$\mathrm{P}_{T}(\Omega \times \mathrm{R})=$ the class of the real functions $v(x, t)$ defined a.e. in $\Omega \times \mathrm{R}$, measurable and T-periodic with respect to $\mathrm{t}$; with $v \in \mathbb{P}_{T}(\Omega \times \mathbb{R})$ and $t \in[0, T] \tilde{v}(t)=v(\cdot, t)$.

We denote by $\mathrm{F}$ the linear map $v \in \mathrm{P}_{T}(\Omega \times \mathrm{R}) \rightarrow \tilde{v}$.

Let $1<p_{1}<\infty, 1<p_{2}<\infty$ and $\mathrm{V}$ be a closed subspace of $W^{n, p_{1}}(\Omega)(n=1,2, \ldots)$ such that $C_{0}^{\infty}(\Omega) \subseteq V$. We do not exclude $V=W^{n, p_{1}}(\Omega)$. Let $\|\cdot\|_{V}$ be a norm equivalent to the one of $W^{n, p_{1}}(\Omega)$ on $\mathrm{V}$.

Set $D^{\alpha}=\frac{\partial^{|\alpha|}}{\partial x_{1}^{\alpha_{1}} \ldots \partial x_{N}^{\alpha_{N}}}$, we consider the normed spaces:

$$
\begin{gathered}
W_{T}^{0}=\left\{v \in \mathbb{P}_{T}(\Omega \times \mathbb{R}) \cap L^{p_{1}}(Q): \text { as } 0<|\alpha| \leq n\right. \text { there exists the weak derivative } \\
\left.D^{\alpha} v \text { with } D^{\alpha} v \in L^{p_{1}}(Q), v(\cdot, t) \in V \text { for a.e.t } \in \mathbb{R}\right\},\|v\|_{0}=\left(\int_{0}^{T}\|v(\cdot, t)\|_{V}^{p_{1}} d t\right)^{1 \backslash p_{1}} \forall v \in W_{T}^{0} ; \\
W_{T}=\left\{v \in W_{T}^{0} \text { : there exists the weak derivative } \frac{\partial v}{\partial t} \text { with } \frac{\partial v}{\partial t} \in L^{p_{2}}(Q)\right\},
\end{gathered}
$$




$$
\begin{gathered}
\|v\|=\|v\|_{0}+\left(\int_{Q}\left|\frac{\partial v}{\partial t}\right|^{p_{2}} d x d t\right)^{1 \backslash p_{2}} \forall v \in W_{T} ; \\
\tilde{W}^{0}=L^{p_{1}}(0, T ; V),\|w\|_{\tilde{W}^{0}}=\left(\int_{0}^{T}\|w(t)\|_{V}^{p_{1}} d t\right)^{1 \backslash p_{1}} \forall w \in \tilde{W}^{0} ; \\
\tilde{W}=\left\{w \in \tilde{W}^{0}: w^{\prime} \in L^{p_{2}}\left(0, T ; L^{p_{2}}(\Omega)\right)\right\},\|w\|_{\tilde{W}}=\|w\|_{\tilde{W}^{0}}+\left(\int_{0}^{T}\left\|w^{\prime}(t)\right\|_{L^{p_{2}}(\Omega)}^{p_{p^{2}}} d t\right)^{1 \backslash p_{2}} \forall w \in \tilde{W} .
\end{gathered}
$$

Remark 1.1. When $p_{1}=p_{2}=p$ we assume

$$
\|v\|=\left(\int_{0}^{T}\|v(\cdot, t)\|_{V}^{p} d t+\int_{Q}\left|\frac{\partial v}{\partial t}\right|^{p} d x d t\right)^{1 \backslash p} \forall v \in W_{T} \text { and }\|w\|_{\tilde{W}}=\left(\int_{0}^{T}\|w(t)\|_{V}^{p} d t+\int_{0}^{T}\left\|w^{\prime}(t)\right\|_{L^{p}(\Omega)}^{p} d t\right)^{1 \backslash p} \forall w \in \tilde{W}
$$

We recall ([16], Chap.23) $\tilde{W}^{0}$ and $\tilde{W}$ are reflexive and separable Banach spaces. It is not difficult to prove that $W_{T}^{0}$ and $W_{T}$ are Banach spaces; the restriction of $\mathrm{F}$ to $W_{T}^{0}\left[\right.$ resp. $\left.W_{T}\right]$ is a norm-preserving linear transformation into $\tilde{W}^{0}[$ resp. $\tilde{W}]$.

Consequently $W_{T}^{0}$ and $W_{T}$ are reflexive and separable spaces.

Remark 1.2. These conclusions hold even if $V=L^{p_{1}}(\Omega)$.In this case $W_{T}^{0}=\mathrm{P}_{T}(\Omega \times \mathbb{R}) \cap L^{p_{1}}(Q)$ and $\|v\|_{0}=\left(\int_{Q}|v|^{p_{1}} d x d t\right)^{1 \backslash p_{1}}$

Let us denote by $\langle\cdot, \cdot\rangle$ the duality between $W_{T}^{*}$ (dual space of $W_{T}$ ) and $W_{T}$, and by " $\partial$ " Fréchet differential operator. Let $\mathrm{A} \sim 0, D_{j} \sim 0(\mathrm{j}=1, \ldots, \mathrm{m} ; m \geq 1)$ and $\mathrm{B}$ be real functionals defined in $W_{T}$ satisfying the conditions

$$
\begin{gathered}
\left(i_{11}\right)\left\{\begin{array}{l}
A \text { is weakly lower semicontinuous in } W_{T} \text { and } C^{1}\left(W_{T}\right), \\
B \text { is weakly continuous in } W_{T} \text { and } C^{1}\left(W_{T}\right), \\
\exists p>1: A(r v)=r^{p} A(v) \text { and } B(r v)=r^{p} B(v) \forall r \geq 0 \text { and } \forall v \in W_{T} ;
\end{array}\right. \\
\left(i_{12}\right)\left\{\begin{array}{l}
D_{j} \text { is weakly continuous in } W_{T} \text { and } C^{1}\left(W_{T}\right), \exists q_{j}>1: \\
D_{j}(r v)=r^{q_{j}} D_{j}(v) \forall r \geq 0 \text { and } \forall v \in W_{T}, q_{1}<\ldots<q_{m} \text { if } m>1 .
\end{array}\right.
\end{gathered}
$$

Let us consider the following problem.

Problem $\left(P^{T}\right)$.Find $u \in W_{T} \backslash\{0\}$ such that

$$
<\partial A(u), v>=\lambda<\partial B(u), v>+\sum_{j=1}^{m}<\partial D_{j}(u), v>\forall v \in W_{T},
$$

where $\lambda$ is a real parameter,

Problem $\left(P^{T}\right)$ is a particular case of Problem $(\mathrm{P})$ studied in ([10], [12]) by using the Lagrange multipliers and the "algebraic" approach which is based on the fibering method [14]. In ([11], [12]) many applications of the results connected to Problem (P) related to nonlinear elliptic systems are present.

In section 2 we considered convenient about Problem $\left(P^{T}\right)$ to state existence theorems (Theorems 2.1-2.3) included in the results of ([4], [7] [8], [10], [12]) whose validity, by the way, depends on $\lambda$. Furthermore we added Propositions 2.1 and 2.2 useful in some concrete cases in order to establish the nonstationarity of the found solutions.

In section 3 we study some evolution PDEs in the cylinder Q, with also nonlocal nonlinearities and with different conditions on 
$\Sigma$. About these problems, whose variational formulation is included in Problem $\left(P^{T}\right)$, we find the existence of one or more weak periodic solutions, giving also some sufficient conditions to their nonstationarity.

It is known that the search for periodic solutions of nonlinear problems has attracted the attention of many researchers. In particular Pohozaev in ([13], [15]) introduced "the separation variables method" for nonlinear equations in which it is possible to find weak periodic solutions in the form $u(x, t)=u_{1}(x) u_{2}(t)$. As far as we know, the methods developed in literature are not applicable in this article.

\section{Existence Theorems Preliminary Results}

As $\lambda \in \mathbb{R}, v \in W_{T}, r \geq 0, j \in\{1, \ldots, m\}$ and $\left\{j_{1}, \ldots, j_{s}\right\} \subseteq\{1, \ldots, m\}\left(j_{1}<\ldots<j_{s}\right.$ if $\left.s>1\right)$ we set:

$$
\begin{gathered}
H_{\lambda}(v)=A(v)-\lambda B(v), E(v)=H_{\lambda}(v)-\sum_{j=1}^{m} D_{j}(v), \tilde{E}(r, v)=E(r v)=r^{p} H_{\lambda}(v)-\sum_{j=1}^{m} r^{q_{j}} D_{j}(v), \\
\Psi(r, v)=\frac{\partial \tilde{E}}{\partial r}(r, v), S_{\lambda}=\left\{v \in W_{T}: H_{\lambda}(v)=1\right\}, V_{\lambda}^{-}=\left\{v \in W_{T}: H_{\lambda}(v)<0\right\}, \\
S\left(D_{j}\right)=\left\{v \in W_{T}: D_{j}(v)=-1\right\}, V^{+}\left(D_{j_{1}}, \ldots, D_{j_{s}}\right)=\left\{v \in W_{T}: D_{j_{1}}(v)+\ldots+D_{j_{s}}(v)>0\right\} .
\end{gathered}
$$

We find the solvability of Problem $\left(P^{T}\right)$ in the cases based on the following assumptions

$$
\begin{gathered}
\left(i_{21}\right) \exists c(\lambda)>0:\|v\|^{p} \leq c(\lambda) H_{\lambda}(v) \forall v \in W_{T} ; \\
\left(i_{22}\right) \exists c(\lambda)>0:\|v\|^{p} \leq c(\lambda) H_{\lambda}(v) \forall v \in V^{+}\left(D_{1}\right) ; \\
\left(i_{23}\right) \exists c(\lambda)>0:\|v\|^{p} \leq c(\lambda) H_{\lambda}(v) \forall v \in V^{+}\left(D_{m}\right) ; \\
\left(i_{24}\right) \exists m_{1} \in\{1, \ldots, m\}: V_{\lambda}^{-} \cap S\left(D_{m_{1}}\right) \text { is nonempty and bounded in } W_{T} .
\end{gathered}
$$

At first we consider the cases in which one of the assumptions $\left(i_{21}\right)-\left(i_{23}\right)$ is present:

$\left(c_{1}\right) m=1, q_{1} \neq p, V^{+}\left(D_{1}\right) \neq \varnothing,\left(i_{22}\right)$ holds;

$\left(c_{2}\right) m>1, q_{1}<p, V^{+}\left(D_{1}\right) \neq \varnothing, D_{j} \leq 0 \forall j \geq 2,\left(i_{22}\right)$ holds;

$\left(c_{3}\right) m>1, q_{m}>p, V^{+}\left(D_{m}\right) \neq \varnothing, D_{j} \leq 0 \forall j \leq m-1,\left(i_{23}\right)$ holds;

$\left(c_{4}\right) m>1, \exists m_{1} \in\{2, \ldots, m\}: q_{m_{1}}<p, D_{j} \geq 0 \forall j \leq m_{1}$ and $D_{j} \leq 0 \forall j>m_{1}$ if $m_{1}<m,\left(i_{21}\right)$ holds;

$\left(c_{5}\right) m>1, \exists m_{1} \in\{1, \ldots, m-1\}: q_{m_{1}}>p, D_{j} \geq 0 \forall j \geq m_{1}$ and $D_{j} \leq 0 \forall j<m_{1}$ if $m_{1}>1,\left(i_{21}\right)$ holds;

$\left(c_{6}\right) m>1, q_{m}<p, D_{j} \geq 0 \forall j<m, D_{m}$ changes sign, $\mathrm{V}^{+}\left(D_{1}, \ldots, D_{m-1}\right)=W_{T} \backslash\{0\},\left(i_{21}\right)$ holds;

$\left(c_{7}\right) m>1, q_{1}>p, D_{1}$ changes sign, $D_{j} \geq 0 \forall j>1, \mathrm{~V}^{+}\left(D_{2}, \ldots, D_{m}\right)=W_{T} \backslash\{0\},\left(i_{21}\right)$ holds.

Let us introduce the open set $\mathscr{Q}$ of the space $W_{T}$ :

$$
\begin{gathered}
\mathbb{Q}=V^{+}\left(D_{1}\right) \text { in }\left(c_{1}\right) \text { and }\left(c_{2}\right), \mathbb{Q}=V^{+}\left(D_{m}\right) \text { in }\left(c_{3}\right), \mathbb{Q}=V^{+}\left(D_{1}, \ldots, D_{m_{1}}\right) \text { in }\left(c_{4}\right), \\
\mathbb{Q}=V^{+}\left(D_{m_{1}}, \ldots, D_{m}\right) \text { in }\left(c_{5}\right), \mathbb{Q}=W_{T} \backslash\{0\} \operatorname{in}\left(c_{6}\right) \text { and }\left(c_{7}\right) .
\end{gathered}
$$

Theorem 2.1 ( [10], Section 2 ). Under assumptions $\left(i_{11}\right)$ and $\left(i_{12}\right)$, in case $\left(c_{1}\right)$ we have: 


$$
\begin{gathered}
\exists v_{0} \in S_{\lambda} \cap \mathbb{Q}: D_{1}\left(v_{0}\right)=\sup \left\{D_{1}(v): v \in S_{\lambda} \cap \mathbb{Q}\right\} \\
\text { with } r_{0}=\left(q_{1} p^{-1} D_{1}\left(v_{0}\right)\right)^{\frac{1}{p-q_{1}}}, u_{0}=r_{0} v_{0} \text { is solution of Problem }\left(P^{T}\right) .
\end{gathered}
$$

When $W_{T}$ is a vector lattice, if $H_{\lambda}(v)=H_{\lambda}(|v|)$ and $D_{1}(v) \leq D_{1}(|v|)\left[\operatorname{resp} \cdot D_{1}(v)=D_{1}(|v|)\right] \forall v \in W_{T}$, then $r_{0}\left|v_{0}\right|$ $\left[\right.$ resp. $r_{0}\left|v_{0}\right|$ and $\left.-r_{0}\left|v_{0}\right|\right]$ is solution $[$ resp. are solutions $]$ of Problem $\left(P^{T}\right)$. Consequently we can assume $v_{0} \geq 0$ i.e. $u_{0} \geq 0$.

When $\mathrm{m}>1$, for any $v \in \mathbb{Q}$ the equation $\Psi(r, v)=0$ has only one positive root $r(v)$ and we have $\frac{\partial \Psi}{\partial r}(r(v), v) \neq 0$.

Evidently

$$
\begin{aligned}
& \text { functionals } r(v) \text { and } \tilde{\tilde{E}}(v)=\tilde{E}(r(v), v)=(r(v))^{p} H_{\lambda}(v)-\sum_{j=1}^{m}(r(v))^{q_{j}} D_{j}(v) \\
& \text { belong to } C^{1}(\mathcal{Q}) \text {. }
\end{aligned}
$$

Theorem 2.2 ([10],Section 2;[12],Section 2).Under assumptions $\left(i_{11}\right)$ and $\left(i_{12}\right)$, in cases $\left(c_{2}\right)-\left(c_{7}\right)$ we have:

$$
\begin{gathered}
\exists v_{0} \in S_{\lambda} \cap \mathbb{Q}: \tilde{\tilde{E}}\left(v_{0}\right)=\inf \left\{\tilde{\tilde{E}}(v): v \in S_{\lambda} \cap \mathbb{Q}\right\} ; \\
\text { with } r_{0}=r\left(v_{0}\right), u_{0}=r_{0} v_{0} \text { is solution of Problem }\left(P^{T}\right) .
\end{gathered}
$$

When $W_{T}$ is a vector lattice, if $H_{\lambda}(v)=H_{\lambda}(|v|)$ and $D_{j}(v)=D_{j}(|v|) \forall v \in W_{T}$ and $\forall j \in\{1, \ldots, m\}, \quad$ then $r_{0}\left|v_{0}\right|$ and $-r_{0}\left|v_{0}\right|$ are solutions of Problem $\left(P^{T}\right)$. Consequently we can assume $v_{0} \geq 0 \quad$ i.e. $u_{0} \geq 0$.

Proposition 2.1. In cases $\left(c_{1}\right)-\left(c_{7}\right)$ let $u_{0}, v_{0}, r_{0}$ be as in Theorems 2.1 and 2.2. If $\tilde{v} \in W_{T}$ is such that $<\partial H_{\lambda}\left(v_{0}\right), \tilde{v}>\neq 0$, then

$$
\sum_{j=1}^{m}\left[<\partial D_{j}\left(u_{0}\right), u_{0}>-p\left(<\partial H_{\lambda}\left(v_{0}\right), \tilde{v}>\right)^{-1}<\partial D_{j}\left(u_{0}\right), r_{0} \tilde{v}>\right]=0 \text { as } m \geq 1
$$

Proof. Let us set $f(s, \tau)=H_{\lambda}\left(s v_{0}+\tau \tilde{v}\right) \forall s>0$ and $\forall \tau \in \mathbb{R}$. We note that $f \in C^{1}(] 0,+\infty[\times \mathbb{R}), \frac{\partial f}{\partial s}(s, \tau)=$ $<\partial H_{\lambda}\left(s v_{0}+\tau \tilde{v}\right), v_{0}>$ and $\left.\frac{\partial f}{\partial \tau}(s, \tau)=<\partial H_{\lambda}\left(s v_{0}+\tau \tilde{v}\right), \tilde{v}>\forall(s, \tau) \in\right] 0,+\infty\left[\times \mathrm{R}\right.$. Since $f(1,0)=1$ and $\frac{\partial f}{\partial \tau}(1,0)=$ $<\partial H_{\lambda}\left(v_{0}\right), \tilde{v}>\neq 0$, there exist $\left.\delta \in\right] 0,1\left[\right.$ and only one function $\tau(s) \in C^{1}(] 1-\delta, 1+\delta[)$ such that $\tau(1)=0$ and $f(s, \tau(s))=1 \quad \forall s \in] 1-\delta, 1+\delta\left[\quad ; \quad\right.$ moreover $\quad \tau^{\prime}(1)=\quad-<\partial H_{\lambda}\left(v_{0}\right), v_{0}>\left(<\partial H_{\lambda}\left(v_{0}\right), \tilde{v}>\right)^{-1}=-p H_{\lambda}\left(v_{0}\right)$ $\left(<\partial H_{\lambda}\left(v_{0}\right), \tilde{v}>\right)^{-1}=-p\left(<\partial H_{\lambda}\left(v_{0}\right), \tilde{v}>\right)^{-1}$.

Let $\left.\left.\delta_{0} \in\right] 0, \delta\right]$ such that $\left.v(s)=s v_{0}+\tau(s) \tilde{v} \in \mathscr{Q} \forall s \in\right] 1-\delta_{0}, 1+\delta_{0}[;$ then

$$
\left.v(s) \in S_{\lambda} \cap \mathbb{Q} \forall s \in\right] 1-\delta_{0}, 1+\delta_{0}[.
$$

In $\left.\left(c_{1}\right)(2.3) \Rightarrow D_{1}(v(s)) \leq D_{1}\left(v_{0}\right)=D_{1}(v(1)) \forall s \in\right] 1-\delta_{0}, 1+\delta_{0}\left[\right.$. Then $\left[\frac{d}{d s} D_{1}(v(s))\right]_{s=1}=0$, from which (2.2) since

$$
\left[\frac{d}{d s} D_{1}(v(s))\right]_{s=1}=<\partial D_{1}\left(v_{0}\right), v_{0}>-p\left(<\partial H_{\lambda}\left(v_{0}\right), \tilde{v}>\right)^{-1}<\partial D_{1}\left(v_{0}\right), \tilde{v}>=
$$




$$
r_{0}^{-q_{1}}\left[<\partial D_{1}\left(u_{0}\right), u_{0}>-p\left(<\partial H_{\lambda}\left(v_{0}\right), \tilde{v}>\right)^{-1}<\partial D_{1}\left(u_{0}\right), r_{0} \tilde{v}>\right] .
$$

In $\left(c_{2}\right)-\left(c_{7}\right) \quad(2.1) \operatorname{and}(2.3) \Rightarrow \quad \tilde{\tilde{E}}(v(s)) \quad \in C^{1}\left(1-\delta_{0}, 1+\delta_{0}[) \quad, \quad(2.3) \Rightarrow \quad \tilde{\tilde{E}}(v(1))=\tilde{\tilde{E}}\left(v_{0}\right) \leq \quad \tilde{\tilde{E}}(v(s))\right.$ $\forall s \in] 1-\delta_{0}, 1+\delta_{0}[$.

Consequently $\left[\frac{d}{d s} \tilde{\tilde{E}}(v(s))\right]_{s=1}=0$, i.e. (2.2). In fact, since

$$
\left.H_{\lambda}(v(s))=1 \text { and } p(r(v(s)))^{p-1}-\sum_{j=1}^{m} q_{j}(r(v(s)))^{q_{j}-1} D_{j}(v(s))=0 \forall s \in\right] 1-\delta_{0}, 1+\delta_{0}[
$$

we have

$$
\begin{gathered}
{\left[\frac{d}{d s} \tilde{\tilde{E}}(v(s))\right]_{s=1}=\left[-\sum_{j=1}^{m} r(v(s))^{q_{j}}<\partial D_{j}(v(s)), v_{0}+\tau^{\prime}(s) \tilde{v}>\right]_{s=1}=} \\
-\sum_{j=1}^{m}\left[r_{0}^{q_{j}}<\partial D_{j}\left(v_{0}\right), v_{0}>-p r_{0}^{q_{j}}\left(<\partial H_{\lambda}\left(v_{0}\right), \tilde{v}>\right)^{-1}<\partial D_{j}\left(v_{0}\right), \tilde{v}>\right]= \\
-\sum_{j=1}^{m}\left[<\partial D_{j}\left(u_{0}\right), u_{0}>-p\left(<\partial H_{\lambda}\left(v_{0}\right), \tilde{v}>\right)^{-1}<\partial D_{j}\left(u_{0}\right), r_{0} \tilde{v}>\right]
\end{gathered}
$$

Let us pass to the cases in which $\left(i_{24}\right)$ is present:

$$
\begin{gathered}
\left(c_{8}\right) m=1, q_{1} \neq p,\left(i_{24}\right) \text { holds }\left(\text { with } m_{1}=1\right) ; \\
\left(c_{9}\right) m>1,\left(i_{24}\right) \text { holds, either } p<q_{1} \text { or } q_{m}<p, D_{j} \leq 0 \text { as } j \neq m_{1} .
\end{gathered}
$$

In $\left(c_{9}\right)$ for any $v \in V_{\lambda}^{-} \cap S\left(D_{m_{1}}\right)$ the equation $\Psi(r, v)=0$ has only one positive root $r(v)$ with $\frac{\partial \Psi}{\partial r}(r(v), v) \neq 0$. Let us set $\tilde{E}(v)=\tilde{E}(r(v), v) \forall v \in V_{\lambda}^{-} \cap S\left(D_{m_{1}}\right)$.

Theorem 2.3 ([10], Section 4 ).Let $\left(i_{11}\right)$ and $\left(i_{12}\right)$ hold. In case $\left(c_{8}\right)$

$$
\begin{gathered}
\exists \underline{v} \in V_{\lambda}^{-} \cap S\left(D_{1}\right): H_{\lambda}(\underline{v})=\inf \left\{H_{\lambda}(v): v \in V_{\lambda}^{-} \cap S\left(D_{1}\right)\right\} ; \\
\text { with } \underline{r}=\left(-p q_{1}^{-1} H_{\lambda}(\underline{v})\right)^{\frac{1}{q_{1}-p}}, \underline{u}=\underline{r} \underline{v} \text { is solution of Problem }\left(P^{T}\right) .
\end{gathered}
$$

In case $\left(c_{9}\right)$

$$
\begin{gathered}
\exists \underline{v} \in V_{\lambda}^{-} \cap S\left(D_{m_{1}}\right): \tilde{\tilde{E}}(\underline{v})=\inf \left\{\tilde{\tilde{E}}(v): v \in V_{\lambda}^{-} \cap S\left(D_{m_{1}}\right)\right\} ; \\
\text { with } \underline{r}=r(\underline{v}), \underline{u}=\underline{r} \underline{v} \text { is solution of Problem }\left(P^{T}\right)
\end{gathered}
$$

When $W_{T}$ is a vector lattice, if $H_{\lambda}(v)=H_{\lambda}(|v|)$ and $D_{j}(v)=D_{j}(|v|) \forall v \in W_{T}$ and $\forall j \in\{1, \ldots, m\}$, then in $\left(c_{8}\right)$ and $\left(c_{9}\right)$ $\underline{r}|\underline{v}|$ and $-\underline{r}|\underline{v}|$ are solutions of Problem $\left(P^{T}\right)$. Consequently we can assume $\underline{v} \geq 0 \quad$ i.e. $\underline{u} \geq 0$.

Proposition 2.2. Let $\underline{u}, \underline{v}, \underline{r}$ be as in Theor. 2.3. In case $\left(c_{8}\right)$ if $\tilde{v} \in W_{T}$ is such that $\left\langle\partial D_{1}(\underline{v}), \tilde{v}\right\rangle \neq 0$, then 


$$
<\partial H_{\lambda}(\underline{u}), \underline{u}>+q_{1}\left(<\partial D_{1}(\underline{v}), \tilde{v}>\right)^{-1}<\partial H_{\lambda}(\underline{u}), \underline{r} \tilde{v}>=0 .
$$

In case $\left(c_{9}\right)$ if $\tilde{v} \in W_{T}$ is such that $\left\langle\partial D_{m_{1}}(\underline{v}), \tilde{v}>\neq 0\right.$, then

$$
\begin{gathered}
\left.\left.\left[<\partial H_{\lambda}(\underline{u}), \underline{u}\right\rangle+q_{m_{1}}\left(\left\langle\partial D_{m_{1}}(\underline{v}), \tilde{v}\right\rangle\right)^{-1}<\partial H_{\lambda}(\underline{u}), \underline{r} \tilde{v}\right\rangle\right]- \\
\left.\sum_{j \neq m_{1}}\left[\left\langle\partial D_{j}(\underline{u}), \underline{u}\right\rangle+q_{m_{1}}\left(\left\langle\partial D_{m_{1}}(\underline{v}), \tilde{v}\right\rangle\right)^{-1}<\partial D_{j}(\underline{u}), \underline{r} \tilde{v}\right\rangle\right]=0 .
\end{gathered}
$$

Proof. As $j_{0} \in\left\{1, m_{1}\right\}$ let us consider the function of $C^{1}(] 0,+\infty[\times \mathbb{R}) \quad f(s, \tau)=D_{j_{0}}(s \underline{v}+\tau \tilde{v})$. Since $f(1,0)=D_{j_{0}}(\underline{v})=-1 \quad$ and $\quad \frac{\partial f}{\partial \tau}(1,0)=\left\langle\partial D_{j_{0}}(\underline{v}), \tilde{v}>\neq 0 \quad\right.$, there exist $\left.\delta \in\right] 0,1[$ and only one function $\tau(s) \in C^{1}([1-\delta, 1+\delta[)$ such that $f(s, \tau(s))=-1 \forall s \in] 1-\delta, 1+\delta[, \tau(1)=0$ and we have

$$
\tau^{\prime}(1)=-<\partial D_{j_{0}}(\underline{v}), \underline{v}>\left(<\partial D_{j_{0}}(\underline{v}), \tilde{v}>\right)^{-1}=-q_{j_{0}} D_{j_{0}}(\underline{v})\left(<\partial D_{j_{0}}(\underline{v}), \tilde{v}>\right)^{-1}=q_{j_{0}}\left(<\partial D_{j_{0}}(\underline{v}), \tilde{v}>\right)^{-1} .
$$

In $\left(c_{8}\right)$, where $j_{0}=1$, with $\left.\left.\delta_{0} \in\right] 0, \delta\right]$ such that $\left.v(s)=s \underline{v}+\tau(s) \tilde{v} \in V_{\lambda}^{-} \forall s \in\right] 1-\delta_{0}, 1+\delta_{0}[$ we have

$$
\left.H_{\lambda}(v(s)) \geq H_{\lambda}(\underline{v})=H_{\lambda}(v(1)) \forall s \in\right] 1-\delta_{0}, 1+\delta_{0}[;
$$

then

$$
\begin{gathered}
0=\left[\frac{d}{d s} H_{\lambda}(v(s))\right]_{s=1}=\left\langle\partial H_{\lambda}(\underline{v}), \underline{v}>+q_{1}\left(<\partial D_{1}(\underline{v}), \tilde{v}>\right)^{-1}<\partial H_{\lambda}(\underline{v}), \tilde{v}>=\right. \\
\underline{r}^{-p}\left[<\partial H_{\lambda}(\underline{u}), \underline{u}>+q_{1}\left(<\partial D_{1}(\underline{v}), \tilde{v}>\right)^{-1}<\partial H_{\lambda}(\underline{u}), \underline{r} \tilde{v}>\right]
\end{gathered}
$$

from which (2.4) follows.

In $\left(c_{9}\right)$, where $j_{0}=m_{1}$, we note that relations $\Psi(\underline{r}, \underline{v})=0$ and $\frac{\partial \Psi}{\partial r}(\underline{r}, \underline{v}) \neq 0$ imply that there exist an open ball $B^{*}$ centered in $\underline{v}$ included in $V_{\lambda}^{-}$and only one functional $r^{*}(v) \in C^{1}\left(B^{*}\right)$ such that $\Psi\left(r^{*}(v), v\right)=0$ for any $v \in B^{*}$. Since $r^{*}(v)=r(v) \forall v \in B^{*} \cap S\left(D_{m_{1}}\right)$, set $\left.\left.\delta_{0} \in\right] 0, \delta\right]$ such that $\left.v(s)=s \underline{v}+\tau(s) \tilde{v} \in B^{*} \forall s \in\right] 1-\delta_{0}, 1+\delta_{0}[$, we have

$$
\left.r^{*}(v(s))=r(v(s)) \forall s \in\right] 1-\delta_{0}, 1+\delta_{0}[.
$$

Then functional

$$
\tilde{\tilde{E}}(v(s))=(r(v(s)))^{p} H_{\lambda}(v(s))-\sum_{j=1}^{m}(r(v(s)))^{q_{j}} D_{j}(v(s))
$$

belongs to $C^{1}(]-\delta_{0}, 1+\delta_{0}[)$.

Taking into account that

$$
\left.D_{m_{1}}(v(s))=-1 \text { and } p(r(v(s)))^{p-1} H_{\lambda}(v(s))-\sum_{j=1}^{m} q_{j}(r(v(s)))^{q_{j}-1} D_{j}(v(s))=0 \quad \forall s \in\right] 1-\delta_{0}, 1+\delta_{0}[\text {, }
$$

we have 


$$
\begin{aligned}
& {\left[\frac{d}{d s} \tilde{\tilde{E}}(v(s))\right]_{s=1}=\underline{r}^{p}\left[\left\langle\partial H_{\lambda}(\underline{v}), \underline{v}\right\rangle+q_{m_{1}}\left(\left\langle\partial D_{m_{1}}(\underline{v}), \tilde{v}\right\rangle\right)^{-1}\left\langle\partial H_{\lambda}(\underline{v}), \tilde{v}\right\rangle\right]-} \\
& \sum_{j \neq m_{1}} \underline{r}^{q_{j}}\left[<\partial D_{j}(\underline{v}), \underline{v}>+q_{m_{1}}\left(<\partial D_{m_{1}}(\underline{v}), \tilde{v}>\right)^{-1}<\partial D_{j}(\underline{v}), \tilde{v}>\right]= \\
& {\left[<\partial H_{\lambda}(\underline{u}), \underline{u}>+q_{m_{1}}\left(<\partial D_{m_{1}}(\underline{v}), \tilde{v}>\right)^{-1}<\partial H_{\lambda}(\underline{u}), \underline{r} \tilde{v}>\right]-} \\
& \sum_{j \neq m_{1}}\left[<\partial D_{j}(\underline{u}), \underline{u}>+q_{m_{1}}\left(<\partial D_{m_{1}}(\underline{v}), \tilde{v}>\right)^{-1}<\partial D_{j}(\underline{u}), \underline{r} \tilde{v}>\right]
\end{aligned}
$$

from which (2.5) since $\tilde{\tilde{E}}(v(s)) \geq \tilde{\tilde{E}}(\underline{v})=\tilde{\tilde{E}}(v(1)) \forall s \in] 1-\delta_{0}, 1+\delta_{0}[$.

\section{Some Applications}

In this section we suppose $N \geq 2$ and set

$|\cdot|$ the Lebesgue measure on $\mathrm{R}$;

$\nu=\left(\nu_{1}, \ldots, \nu_{N}\right)=$ the outward orthogonal unitary vector to $\partial \Omega$;

$\mathrm{P}_{T}(\mathrm{R})=$ the class of the real functions defined a.e.in $\mathrm{R}$, measurable and T-periodic;

$\forall \varphi \in \mathbb{P}_{T}(\mathrm{R}) \cap C^{0}(\mathrm{R}) \operatorname{supp}_{[0, T]} \varphi=$ the support of the restriction of $\varphi$ to $[0, T]$.

We warn that the weak continuity in $W_{T}$ of the functionals $B$ and $D_{j}$ present in the applications can be easily proved by using embedding Sobolev theorems [1], a compactness lemma ([6], Theor.5.1 page 58) and the isomorphism F (section 1).

We add the following clarification:

$$
\left(c^{*}\right)\left[\begin{array}{l}
\text { With } \left.t_{0} \in\right] 0, T\left[\text { and } \varepsilon_{0}>0\left[\text { resp.with } \varepsilon_{0}>0\right] \text { we suppose } 0<t_{0}-\varepsilon_{0} \text { and } t_{0}+\varepsilon_{0}<T\left[\text { resp. } \varepsilon_{0}<T-\varepsilon_{0}\right] .\right. \\
\text { Additionally as } 0<\varepsilon<\varepsilon_{0} / 2 \text { we denote by } \omega_{\varepsilon} \text { a nonnegative function belonging to } \mathrm{P}_{T}(\mathrm{R}) \cap \mathrm{C}^{\infty}(\mathrm{R}) \\
\text { such that } \left.\operatorname{supp}_{[0, T]} \omega_{\varepsilon} \subseteq\right] t_{0}-2 \varepsilon, t_{0}+2 \varepsilon\left[\text { and } \omega_{\varepsilon}=1 \text { in }\left[t_{0}-\varepsilon, t_{0}+\varepsilon\right]\right. \\
r \text { resp. supp } \omega_{\varepsilon} \subseteq[0,2 \varepsilon[\cup] T-2 \varepsilon, T] \text { and } \omega_{\varepsilon}=1 \text { in }[0, \varepsilon] \cup[T-\varepsilon, T]
\end{array}\right] .
$$

Application 3.1 (connected to Theor.2.1). Let us assume in the definition (1.1) of $W_{T} n=1$ and $V=W_{0}^{1, p_{1}}(\Omega)$, then

$$
\|v\|=\left(\int_{Q}\left|\frac{\partial v}{\partial t}\right|^{p_{2}} d x d t\right)^{1 / p_{2}}+\left(\int_{Q}|\nabla v|^{p_{1}} d x d t\right)^{1 / p_{1}} \forall v \in W_{T}
$$

and let us set as any $v \in W_{T}$

$$
\begin{gathered}
A(v)=p^{-1}\left[\left(\left.\int_{Q}\left|\frac{\partial v}{\partial t}\right|^{p_{2}} d x d t\right|^{\frac{p}{p_{2}}}+\left(\int_{Q} a(x, t)|\nabla v|^{p_{1}} d x d t\right)^{\frac{p}{p_{1}}}\right],\right. \\
B(v)=p^{-1}\left(\int_{Q} b(x, t)|v|^{p_{1}} d x d t\right)^{\frac{p}{p_{1}}}, \quad D_{1}(v)=q_{1}^{-1}\left|\int_{Q} d_{1}(x, t) v d x d t\right|^{q_{1}},
\end{gathered}
$$

where 


$$
\begin{gathered}
1<p_{1} \leq p, 1<p_{2} \leq p, 1<q_{1}, q_{1} \neq p ; a, b \in\left(\mathrm{P}_{T}(\Omega \times \mathbb{R}) \cap L^{\infty}(Q)\right) \backslash\{0\}, a(x, t) \geq a_{0} \text { and } 0 \leq b(x, t) \leq b_{0} \\
\text { a.e. in } Q\left(a_{0}, b_{0}=\text { const. }>0\right), d_{1} \in\left(\mathbb{P}_{T}(\Omega \times \mathbb{R}) \cap L^{p_{1}^{\prime}}(Q)\right) \backslash\{0\}\left(p_{1}^{\prime}=p_{1} /\left(p_{1}-1\right)\right) \text {. }
\end{gathered}
$$

Problem $\left(P^{T}\right)$ becomes:

Find $u \in W_{T} \backslash\{0\}$ such that

$$
\left(\int_{Q}\left|\frac{\partial u}{\partial t}\right|^{p_{2}} d x d t\right)^{\frac{p}{p_{2}}-1} \int_{Q}\left|\frac{\partial u}{\partial t}\right|^{p_{2}-2} \frac{\partial u}{\partial t} \frac{\partial v}{\partial t} d x d t+\left(\int_{Q} a(x, t)|\nabla u|^{p_{1}} d x d t\right)^{\frac{p}{p_{1}}-1} \int_{Q} a(x, t)|\nabla u|^{p_{1}-2} \nabla u \nabla v d x d t=
$$

$\lambda\left(\left.\int_{Q} b(x, t)|u|^{p_{1}} d x d t\right|^{\frac{p}{p_{1}}-1} \int_{Q} b(x, t)|u|^{p_{1}-2} u v d x d t+\left.\left|\int_{Q} d_{1}(x, t) u d x d t\right|\right|_{Q} ^{q_{1}-2}\left(\int_{Q} d_{1}(x, t) u d x d t\right) \int_{Q} d_{1}(x, t) v d x d t \quad \forall v \in W_{T}\right.$.

Each solution $u$ of (3.2) is for definition a weak solution of the problem:

$$
\begin{gathered}
-\left(\left.\int_{Q}\left|\frac{\partial u}{\partial t}\right|^{p_{2}} d x d t\right|^{\frac{p}{p_{2}}-1} \frac{\partial}{\partial t}\left(\left|\frac{\partial u}{\partial t}\right|^{p_{2}-2} \frac{\partial u}{\partial t}\right)-\left.\left.\left|\int_{Q} a(x, t)\right| \nabla u\right|^{p_{1}} d x d t\right|^{\frac{p}{p_{1}}-1} \operatorname{div}\left(a(x, t)|\nabla u|^{p_{1}-2} \nabla u\right)=\right. \\
\lambda\left(\int_{Q} b(x, t)|u|^{p_{1}} d x d t\right)^{\frac{p}{p_{1}}-1} b(x, t)|u|^{p_{1}-2} u+\left.\left|\int_{Q} d_{1}(x, t) u d x d t\right|\right|_{Q} ^{p_{1}-2}\left(\int_{Q} d_{1}(x, t) u d x d t\right) d_{1}(x, t) \text { in } Q, \\
u=0 \text { on } \Sigma, u(x, 0)=u(x, T) \text { and } \frac{\partial u}{\partial t}(x, 0)=\frac{\partial u}{\partial t}(x, T) \text { on } \Omega .
\end{gathered}
$$

Evidently

$$
V^{+}\left(D_{1}\right) \neq \varnothing
$$

Let $\lambda^{*}$ and $z^{*}$ be the first eigenvalue and the first eigenfunction of the problem:

$$
z \in W_{0}^{1, p_{1}}(\Omega):-a_{0} \operatorname{div}\left(|\nabla z|^{p_{1}-2} \nabla z\right)=\theta b_{0}|z|^{p_{1}-2} z \quad \text { in } \Omega .
$$

We remember that [3] $z^{*}>0$ in $\Omega$ and

$$
\lambda^{*}=\left(a_{0} \int_{\Omega}\left|\nabla z^{*}\right|^{p_{1}} d x\right)\left(b_{0} \int_{\Omega}\left(z^{*}\right)^{p_{1}} d x\right)^{-1} \leq\left(a_{0} \int_{\Omega}|\nabla z|^{p_{1}} d x\right)\left(b_{0} \int_{\Omega}|z|^{p_{1}} d x\right)^{-1} \forall z \in W_{0}^{1, p_{1}}(\Omega) \backslash\{0\} .
$$

(3.5) implies that

$$
b_{0} \int_{\Omega}|v(x, t)|^{p_{1}} d x \leq\left(\lambda^{*}\right)^{-1} a_{0} \int_{\Omega}|\nabla v(x, t)|^{p_{1}} d x \text { a.e. in }[0, T] \forall v \in W_{T} \text {; }
$$

then as $0<\lambda<\left(\lambda^{*}\right)^{\frac{p}{p_{1}}}$ 


$$
\left(a_{0} \int_{Q}|\nabla v|^{p_{1}} d x d t\right)^{\frac{p}{p_{1}}}-\lambda\left(b_{0} \int_{Q}|v|^{p_{1}} d x d t\right)^{\frac{p}{p_{1}}} \geq\left(1-\lambda\left(\lambda^{*}\right)^{-\frac{p}{p_{1}}}\right)\left(a_{0} \int|\nabla v|_{Q}^{p_{1}} d x d t\right)^{\frac{p}{p_{1}}} \forall v \in W_{T}
$$

from which

$$
H_{\lambda}(v)=A(v)-\lambda B(v) \geq p^{-1} \min \left\{1, a_{0}^{\frac{p}{p_{1}}}\right\}\left(1-\lambda\left(\lambda^{*}\right)^{-\frac{p}{p_{1}}}\right) 2^{-p}\|v\|^{p} \forall v \in W_{T}
$$

Then, since

$$
\text { as } \lambda \leq 0 \quad\|v\|^{p} \leq p 2^{p} H_{\lambda}(v) \quad \forall v \in W_{T}
$$

we have

$$
\left.\left(i_{21}\right), \text { in particular }\left(i_{22}\right), \text { holds if } \lambda \in\right]-\infty,\left(\lambda^{*}\right)^{\frac{p}{p_{1}}}[\text {. }
$$

Relations (3.4),(3.7) and Theor.2.1 let us to state the following proposition.

Proposition 3.1. Under conditions (3.1), with $\lambda$ as in (3.7) problem (3.3) has at least two weak solutions $u_{0}$ and $-u_{0}$ $\left(u_{0}=r_{0} v_{0}, r_{0}=\right.$ const. $>0, v_{0} \in S_{\lambda} \cap V^{+}\left(D_{1}\right)$ ).

Remark 3.1. If $d_{1} \geq 0$, we have $D_{1}(v) \leq D_{1}(|v|) \forall v \in W_{T}$. Then, since $H_{\lambda}(v)=H_{\lambda}(|v|) \forall v \in W_{T}$, it results in $u_{0} \geq 0$. Additionally, when $a(x, t) \equiv a_{0}$ and $b(x, t) \equiv b_{0}$ in $Q$, since $z^{*} \in V^{+}\left(D_{1}\right)$ and $H_{\lambda}\left(z^{*}\right) \leq 0$ as $\lambda \geq\left(\lambda^{*}\right)^{\frac{p}{p_{1}}},\left(i_{22}\right)$ holds if and only if $\lambda \in]-\infty,\left(\lambda^{*}\right)^{\frac{p}{p_{1}}}[$.

Proposition 3.2. Let $p_{2} \leq N p_{1} /\left(N-p_{1}\right)$ if $N>p_{1}$. If there exist a measurable set $I \subseteq[0, T]$ with $|I|_{1}>0$, a limit point $t_{0}$ of $I$ and $g \in L^{p_{0}}(\Omega)\left(p_{0}=\min \left\{p_{1}^{\prime}, p_{2}^{\prime}\right\}, p_{i}^{\prime}=p_{i} /\left(p_{i}-1\right)\right)$ such that $\lim _{\substack{t \rightarrow t_{j} \\ t \in I}} d_{1}(x, t)=0$ a.e. in $\Omega$ and $\left|d_{1}(x, t)\right| \leq g(x)$ a.e. in $\Omega \times I$, then $u_{0}$ is nonstationary.

Proof. The additional assumption on $p_{2}$ implies that

$$
W_{0}^{1, p_{1}}(\Omega) \subseteq L^{p_{2}}(\Omega) \text { with continuous embedding. }
$$

Reasoning by contradiction, let $\frac{\partial u_{0}}{\partial t} \equiv 0 \operatorname{in} Q$. Set $\omega(t)=\int_{\Omega} d_{1}(x, t) u_{0} d x$ a.e. in $[0, T]$, a Lebesgue theorem assures that

$$
\lim _{\substack{t \rightarrow t \\ t \in Y}} \omega(t)=0
$$

Let $\varphi \in \mathbb{P}_{T}(\mathbb{R}) \cap C^{\infty}(\mathbb{R})$ with $\varphi \geq 0$ and $\left.\operatorname{supp}_{[0, T]} \varphi \subset\right] 0, T\left[\right.$. Since by (3.8) $\varphi u_{0} \in W_{T}$, from (3.2) with $u=u_{0}$ and $v=\varphi u_{0}$ we get

$$
\left(\left.\int_{Q} a(x, t)\left|\nabla u_{0}\right|^{p_{1}} d x d t\right|^{\frac{p}{p_{1}}-1} \int_{Q} a(x, t)\left|\nabla u_{0}\right|^{p_{1}} \varphi d x d t-\lambda\left(\left.\int_{Q} b(x, t)\left|u_{0}\right|^{p_{1}} d x d t\right|^{\frac{p}{p_{1}}-1} \int_{Q} b(x, t)\left|u_{0}\right|^{p_{1}} \varphi d x d t=\right.\right.
$$




$$
\left|\int_{Q} d_{1}(x, t) u_{0} d x d t\right|^{q_{1}-2}\left(\int_{Q} d_{1}(x, t) u_{0} d x d t\right)\left(\int_{Q} d_{1}(x, t) u_{0} \varphi d x d t\right) .
$$

Let us add that

$$
\text { the left side of (3.10) is } \geq(T)^{-1} \delta \int_{0}^{T} \varphi d t
$$

where

$$
\begin{gathered}
\delta=\left(\int_{Q} a_{0}\left|\nabla u_{0}\right|^{p_{1}} d x d t\right)^{\frac{p}{p_{1}}}>0 \text { if } \lambda \leq 0, \\
\delta=\left(\int_{Q} a_{0}\left|\nabla u_{0}\right|^{p_{1}} d x d t\right)^{\frac{p}{p_{1}}}-\lambda\left(\int_{Q} b_{0}\left|u_{0}\right|^{p_{1}} d x d t\right)^{\frac{p}{p_{1}}}>0(\text { from }(3.6)) \text { if } 0<\lambda<\left(\lambda^{*}\right)^{\frac{p}{p_{1}}} .
\end{gathered}
$$

Since $u_{0} \in V^{+}\left(D_{1}\right) \Rightarrow \int_{0}^{T} \omega d t \neq 0$, from (3.10),(3.11) we get

$$
\omega(t) \geq \text { const. }>0 \text { if } \int_{0}^{T} \omega d t>0, \omega(t) \leq \text { const. }<0 \text { if } \int_{0}^{T} \omega d t<0 \text { a.e.in }[0, T] .
$$

Then (3.12) contradicts (3.9).

Application 3.2 (connected to Theor.2.1 and Theor.2.3 (case $\left(\mathrm{c}_{8}\right)$ )). Let us assume in the definition (1.1) of $W_{T} \quad p_{1}=p_{2}=p, n=1$ and $V=W^{1, p}(\Omega)$, then

$$
\|v\|=\left(\int_{Q}\left|\frac{\partial v}{\partial t}\right|^{p} d x d t+\int_{Q}|v|^{p} d x d t+\int_{Q}|\nabla v|^{p} d x d t\right)^{1 / p} \forall v \in W_{T},
$$

and let us set as any $v \in W_{T}$

$$
\begin{gathered}
A(v)=p^{-1}\left[\int_{Q}\left|\frac{\partial v}{\partial t}\right|^{p} d x d t+\int_{Q} a(x, t)|\nabla v|^{p} d x d t\right], \\
B(v)=p^{-1} \int_{Q} b(x, t)|v|^{p} d x d t, \quad D_{1}(v)=q_{1}^{-1} \int_{Q} d_{1}(x, t)|v|^{q_{1}} d x d t,
\end{gathered}
$$

where

$$
\begin{gathered}
1<q_{1}<p ; a, b, d_{1} \in\left(\mathbb{P}_{T}(\Omega x \mathbb{R}) \cap L^{\infty}(Q)\right) \backslash\{0\}, a(x, t) \geq a_{0} \text { and } 0<b(x, t) \leq b_{0} \\
\text { a.e.in } Q\left(a_{0}, b_{0}=\text { const. }>0\right) .
\end{gathered}
$$

Problem $\left(P^{T}\right)$ becomes:

Find $u \in W_{T} \backslash\{0\}$ such that 
$\int_{Q}\left|\frac{\partial u}{\partial t}\right|^{p-2} \frac{\partial u}{\partial t} \frac{\partial v}{\partial t} d x d t+\int_{Q} a(x, t)|\nabla u|^{p-2} \nabla u \nabla v d x d t=\lambda \int_{Q} b(x, t)|u|^{p-2} u v d x d t+\int_{Q} d_{1}(x, t)|u|^{q_{1}-2} u v d x d t \quad \forall v \in W_{T} \cdot$

Each solution $u$ of (3.14) is for definition a weak solution of the problem:

$$
\begin{gathered}
-\frac{\partial}{\partial t}\left(\left|\frac{\partial u}{\partial t}\right|^{p-2} \frac{\partial u}{\partial t}\right)-\operatorname{div}\left(a(x, t)|\nabla u|^{p-2} \nabla u\right)=\lambda b(x, t)|u|^{p-2} u+d_{1}(x, t)|u|^{q_{1}-2} u \text { in } Q \\
a(x, t)|\nabla u|^{p-2} \frac{\partial u}{\partial \nu}=0 \text { on } \Sigma, u(x, 0)=u(x, T) \text { and } \frac{\partial u}{\partial t}(x, 0)=\frac{\partial u}{\partial t}(x, T) \text { on } \Omega .
\end{gathered}
$$

Let us introduce the conditions

$$
\begin{aligned}
& d_{1}^{+}=\max \left\{d_{1}, 0\right\} \smile 0 \text { in } \mathrm{Q}, \\
& \int_{Q} d_{1}(x, t) d x d t<0 .
\end{aligned}
$$

Evidently

$$
(3.16) \Rightarrow V^{+}\left(D_{1}\right) \neq \varnothing,(3.17) \Rightarrow V_{\lambda}^{-} \cap S\left(D_{1}\right) \neq \varnothing \forall \lambda>0 .
$$

Proposition 3.3.Under conditions (3.13) (with $\mathrm{p}>1$ and not necessarily $\left.>\mathrm{q}_{1}\right)$, $\left(\mathrm{i}_{21}\right)$ holds if $\lambda<0$.

Proof. Let $\lambda<0$. Reasoning by contradiction, as any $k \in \mathbb{N}$ there exists $v_{k} \in W_{T}$ such that

$$
H_{\lambda}\left(v_{k}\right)<k^{-1}\left\|v_{k}\right\|^{p}
$$

Then with $w_{k}=\left\|v_{k}\right\|^{-1} v_{k}$ we have

$$
\int_{Q}\left|\frac{\partial w_{k}}{\partial t}\right|^{p} d x d t+\int_{Q} a(x, t)\left|\nabla w_{k}\right|^{p} d x d t-\lambda \int_{Q} b(x, t)\left|w_{k}\right|^{p} d x d t \leq p k^{-1}
$$

moreover there exists $w \in W_{T}$ such that (within a subsequence) $w_{k} \rightarrow$ w weakly in $W_{T}$.

Consequently

$$
\begin{gathered}
\int_{Q}\left|\frac{\partial w}{\partial t}\right|^{p} d x d t=\lim _{k \rightarrow+\infty} \int_{Q}\left|\frac{\partial w_{k}}{\partial t}\right|^{p} d x d t=0, \int_{Q} a(x, t)|\nabla w|^{p} d x d t=\lim _{k \rightarrow+\infty} \int_{Q} a(x, t)\left|\nabla w_{k}\right|^{p} d x d t=0 \\
\int_{Q} b(x, t)|w|^{p} d x d t=\lim _{k \rightarrow+\infty} \int_{Q} b(x, t)\left|w_{k}\right|^{p} d x d t=0,
\end{gathered}
$$

from which $w_{k} \rightarrow 0$ strongly in $W_{T}$ and the contradiction $1=\lim _{k \rightarrow+\infty}\left\|w_{k}\right\|=0$.

Proposition 3.4. Under conditions (3.13), (3.16) and (3.17), there exists $\delta_{1}^{*}>0$ satisfying the condition

$$
\forall \lambda \in\left[0, \delta_{1}^{*}\right] \exists c(\lambda)>0: \int_{Q}\left|\frac{\partial v}{\partial t}\right|^{p} d x d t+a_{0} \int_{Q}|\nabla v|^{p} d x d t-\lambda b_{0} \int_{Q}|v|^{p} d x d t \geq c(\lambda)\|v\|^{p} \forall v \in V^{+}\left(D_{1}\right)
$$

Consequently $\left(i_{22}\right)$ holds if $\lambda \in\left[0, \delta_{1}^{*}\right]$.

Proof. Reasoning by contradiction, as any $k \in \mathbb{N}$ there exist $v_{k} \in V^{+}\left(D_{1}\right)$ and $\lambda_{k} \in\left[0, k^{-1}\right]$ such that 


$$
\int_{Q}\left|\frac{\partial v_{k}}{\partial t}\right|^{p} d x d t+a_{0} \int_{Q}\left|\nabla v_{k}\right|^{p} d x d t<\lambda_{k} b_{0} \int_{Q}\left|v_{k}\right|^{p} d x d t+k^{-1}\left\|v_{k}\right\|^{p} .
$$

Then with $w_{k}=\left\|v_{k}\right\|^{-1} v_{k}$ there exists $w \in W_{T}$ such that (within a subsequence)

$$
\begin{gathered}
w_{k} \rightarrow \text { w weakly in } W_{T}, \\
\int_{Q}\left|\frac{\partial w}{\partial t}\right|^{p} d x d t=\lim _{k \rightarrow+\infty} \int_{Q}\left|\frac{\partial w_{k}}{\partial t}\right|^{p} d x d t=0, \int_{Q}|\nabla w|^{p} d x d t=\lim _{k \rightarrow+\infty} \int_{Q}\left|\nabla w_{k}\right|^{p} d x d t=0, \\
\int_{Q} d_{1}(x, t)|w|^{q_{1}} d x d t=\lim _{k \rightarrow+\infty} \int_{Q} d_{1}(x, t)\left|w_{k}\right|^{q_{1}} d x d t \geq 0,
\end{gathered}
$$

from which since (3.17) we get $w \equiv 0$ in $Q$. Then $w_{k} \rightarrow 0$ strongly in $W_{T}$ and the contradiction $1=\lim _{k \rightarrow+\infty}\left\|w_{k}\right\|=0$.

Proposition 3.5. Under conditions (3.13) and (3.17), there exists $\delta_{2}^{*}>0$ such that $\left(i_{24}\right)$ holds if $\left.\left.\lambda \in\right] 0, \delta_{2}^{*}\right]$.

Proof. Reasoning by contradiction, as any $k \in \mathbb{N}$ there exist $\left.\left.\lambda_{k} \in\right] 0, k^{-1}\right]$ and $\left(v_{k, h}\right)_{h \in \mathbb{N}} \subseteq W_{T}$ such that

$$
\begin{gathered}
D_{1}\left(v_{k, h}\right)=-1, \\
H_{\lambda_{k}}\left(v_{k, h}\right)<0, \\
\lim _{h \rightarrow+\infty}\left\|v_{k, h}\right\|^{p}=+\infty .
\end{gathered}
$$

Relation (3.20) implies there exists $\left(h_{k}\right)_{k \in \mathbb{N}} \subseteq \mathbb{N}$ strictly increasing such that

$$
\lim _{k \rightarrow+\infty}\left\|v_{k, h_{k}}\right\|^{p}=+\infty .
$$

Set $w_{k}=\left\|v_{k, h_{k}}\right\|^{-1} v_{k, h_{k}}$, from (3.18), (3.19) we get

$$
\begin{aligned}
& \int_{Q} d_{1}(x, t)\left|w_{k}\right|^{q_{1}} d x d t=-q_{1}|| v_{k, h_{k}} \|^{-q_{1}}, \\
& \int_{Q}\left|\frac{\partial w_{k}}{\partial t}\right|^{p} d x d t+\int_{Q} a(x, t)\left|\nabla w_{k}\right|^{p} d x d t<\lambda_{k} \int_{Q} b(x, t)\left|w_{k}\right|^{p} d x d t .
\end{aligned}
$$

Let $w \in W_{T}$ such that (within a subsequence) $w_{k} \rightarrow$ weakly in $W_{T}$.

From (3.21), (3.22) we get

$$
\begin{gathered}
\int_{Q} d_{1}(x, t)|w|^{q_{1}} d x d t=\lim _{k \rightarrow+\infty} \int_{Q} d_{1}(x, t)\left|w_{k}\right|^{q_{1}} d x d t=0, \int_{Q}\left|\frac{\partial w}{\partial t}\right|^{p} d x d t=\lim _{k \rightarrow+\infty} \int_{Q}\left|\frac{\partial w_{k}}{\partial t}\right|^{p} d x d t=0, \\
\int_{Q} a(x, t)|\nabla w|^{p} d x d t=\lim _{k \rightarrow+\infty} \int_{Q} a(x, t)\left|\nabla w_{k}\right|^{p} d x d t=0,
\end{gathered}
$$

from which since (3.17) $w_{k} \rightarrow 0$ strongly in $W_{T}$ and the contradiction $1=\lim _{k \rightarrow+\infty}\left\|w_{k}\right\|=0$. 
Propositions 3.3-3.5, Theorems 2.1 and 2.3 (case $\mathrm{c}_{8}$ ) allow the following proposition.

Proposition 3.6. Under assumptions (3.13) we have:

when (3.16) and (3.17) hold, with $\left.\lambda \in]-\infty, \delta_{1}^{*}\right]$ problem (3.15) has at least two weak solutions $u_{0} \geq 0$ and $-u_{0}\left(u_{0}=r_{0} v_{0}\right.$, $r_{0}=$ const. $\left.>0, v_{0} \in S_{\lambda} \cap V^{+}\left(D_{1}\right)\right)$;

when (3.17) holds, with $\left.\lambda \in] 0, \delta_{2}^{*}\right]$ problem (3.15) has at least two weak solutions $\underline{u} \geq 0$ and $-\underline{u}$ ( $\underline{u}=\underline{r} \underline{v}$, $\underline{r}=$ const. $\left.>0, \underline{v} \in V_{\lambda}^{-} \cap S\left(D_{1}\right)\right)$.

Consequently, when (3.16) and (3.17) hold, with $\left.\lambda \in] 0, \min \left\{\delta_{1}^{*}, \delta_{2}^{*}\right\}\right]$ problem (3.15) has at least four different weak solutions.

Proposition 3.7.Let (3.13), (3.16) and (3.17) hold. Let $\left.\lambda \in]-\infty, \delta_{1}^{*}\right]$. If there exists a measurable set $I \subseteq[0, T]$ with $|I|_{1}>0$ such that $d_{1}(x, t) \leq 0$ a.e. in $\Omega \times I$, then $\mathrm{u}_{0}$ is nonstationary.

Proof. Reasoning by contradiction let $\frac{\partial u_{0}}{\partial t} \equiv 0$ in $Q$. With $\omega(t)=\int_{\Omega} d_{1}(x, t)\left(u_{0}\right)^{q_{1}} d x$ a.e. in $[0, T]$ we have

$$
\omega(t) \leq 0 \text { a.e. in } I
$$

Let $\varphi \in \mathbb{P}_{T}(\mathbb{R}) \cap C^{\infty}(\mathbb{R})$ with $\varphi \geq 0$ and $\left.\operatorname{supp}_{[0, T]} \varphi \subset\right] 0, T\left[\right.$. Setting in (3.14) $u=u_{0}$ and $v=\varphi u_{0} \in W_{T}$, we have

$$
\int_{Q} a(x, t)\left|\nabla u_{0}\right|^{p} \varphi d x d t-\lambda \int_{Q} b(x, t)\left(u_{0}\right)^{p} \varphi d x d t=\int_{0}^{T} \omega \varphi d t
$$

from which

$$
(T)^{-1} \delta \int_{0}^{T} \varphi d t \leq \int_{0}^{T} \omega \varphi d t
$$

where

$$
\begin{gathered}
\delta=a_{0} \int_{Q}\left|\nabla u_{0}\right|^{p} d x d t>0\left(\text { since } \mathrm{u}_{0} \in V^{+}\left(D_{1}\right)\right) \text { if } \lambda<0, \\
\delta=a_{0} \int_{Q}\left|\nabla u_{0}\right|^{p} d x d t-\lambda b_{0} \int_{Q}\left(u_{0}\right)^{p} d x d t>0 \text { (since Prop.3.4) if } \lambda \in\left[0, \delta_{1}^{*}\right] .
\end{gathered}
$$

Then $\omega(t) \geq(T)^{-1} \delta$ a.e.in $[0, T]$, and this contradicts (3.23).

Proposition 3.8. Let (3.13) and (3.17) hold. Let $\left.\lambda \in] 0, \delta_{2}^{*}\right]$. Let one of the following conditions holds:

There exists a measurable set $I \subseteq[0, T]$ with $|I|_{1}>0$ such that $d_{1}(x, t) \geq 0$ a.e. in $\Omega \times I$;

There exist a measurable set $I \subseteq[0, T]$ with $|I|_{1}>0, c_{0}>0$ and a limit point $t_{0}$ of $I$ such that $b(x, t) \geq c_{0}$ a.e. in $\Omega \times I$, $\lim _{\substack{t \rightarrow t_{0} \\ t \in \rho}} d_{1}(x, t)=0$ a.e.in $\Omega$;

There exist $\left.t_{0} \in\right] 0, T\left[\right.$ and $\varepsilon_{0}>0\left[\right.$ resp.there exists $\left.\varepsilon_{0}>0\right]$ as in $\left(c^{*}\right), \eta>0, h>1$ and $\theta \in \mathbb{P}_{T}(\mathbb{R}) \cap W^{1, p}(] 0, T[)$, with $\theta(t)>0 \quad \forall t \in\left[t_{0}-\varepsilon_{0}, t_{0}+\varepsilon_{0}\right] \backslash\left\{t_{0}\right\} \quad[$ resp. $\left.\forall t \in] 0, \varepsilon_{0}\right] \cup\left[T-\varepsilon_{0}, T[]\right.$ and $\theta\left(t_{0}\right)=0[$ resp. $\theta(0)=0], \quad$ such that 
$b(x, t) \geq \theta(t)$ and $-\eta(\theta(t))^{h} \leq d_{1}(x, t)<0$ a.e. in $\Omega \times\left[t_{0}-\varepsilon_{0}, t_{0}+\varepsilon_{0}\right]\left[\right.$ resp.a.e.in $\left.\Omega \times\left(\left[0, \varepsilon_{0}\right] \cup\left[T-\varepsilon_{0}, T\right]\right)\right]$.

Then $\underline{u}$ is nonstationary.

Proof. Under condition (3.24) [resp. (3.25)], reasoning by contradiction let $\frac{\partial \underline{u}}{\partial t} \equiv 0$ in $Q$.

Let $\varphi \in \mathbb{P}_{T}(\mathbb{R}) \cap C^{\infty}(\mathbb{R})$ with $\left.\operatorname{supp}_{[0, T]} \varphi \subset\right] 0, T\left[\right.$. Relation (3.14) with $u=\underline{u}$ and $v=\varphi \in W_{T}$ becomes

from which

$$
\int_{0}^{T}\left(\int_{\Omega} d_{1}(x, t)(\underline{u})^{q_{1}-1} d x\right) \varphi d t=-\lambda \int_{0}^{T}\left(\int_{\Omega} b(x, t)(\underline{u})^{p-1} d x\right) \varphi d t
$$

and then the contradiction

$$
\int_{\Omega} d_{1}(x, t)(\underline{u})^{q_{1}-1} d x=-\lambda \int_{\Omega} b(x, t)(\underline{u})^{p-1} d x \text { a.e. in }[0, T]
$$

$$
\begin{gathered}
0 \leq \int_{\Omega} d_{1}(x, t)(\underline{u})^{q_{1}-1} d x<0 \text { a.e.in } I \\
{\left[\operatorname{resp} \cdot \int_{\Omega} d_{1}(x, t)(\underline{u})^{q_{1}-1} d x \leq-\lambda c_{0} \int_{\Omega}(\underline{u})^{p-1} d x \text { a.e.in I and } \lim _{\substack{t \rightarrow t_{f} \\
t \in I}} \int_{\Omega} d_{1}(x, t)(\underline{v})^{q_{1}-1} d x=0\right] .}
\end{gathered}
$$

Under condition (3.26), according to Prop.2.2 it is sufficiently to prove that If $\frac{\partial \underline{u}}{\partial t} \equiv 0$ in $Q$, then there exists $\tilde{v} \in W_{T}$ such that $\left\langle\partial D_{1}(\underline{v}), \tilde{v}>\neq 0\right.$ and

$$
<\partial H_{\lambda}(\underline{u}), \underline{u}>+q_{1}\left(<\partial D_{1}(\underline{v}), \tilde{v}>\right)^{-1}<\partial H_{\lambda}(\underline{u}), \underline{r} \tilde{v}>\neq 0 .
$$

Let $\omega_{\varepsilon}$ be as in $\left(c^{*}\right)$. Since $\theta \omega_{\varepsilon} \in W_{T}$, we have

$$
\begin{gathered}
<\partial D_{1}(\underline{v}), \theta \omega_{\varepsilon}>=\int_{Q} d_{1}(x, t)(\underline{v})^{q_{1}-1} \theta \omega_{\varepsilon} d x d t<0 \\
<\partial D_{1}(\underline{v}), \theta \omega_{\varepsilon}>\geq-\eta \int_{Q}(\underline{v})^{q_{1}-1} \theta^{h+1} \omega_{\varepsilon} d x d t=-\eta\left(\int_{\Omega}(\underline{v})^{q_{1}-1} d x\right) \int_{0}^{T} \theta^{h+1} \omega_{\varepsilon} d t .
\end{gathered}
$$

Then

$$
\begin{gathered}
<\partial H_{\lambda}(\underline{u}), \underline{u}>+q_{1}\left(<\partial D_{1}(\underline{v}), \theta \omega_{\varepsilon}>\right)^{-1}<\partial H_{\lambda}(\underline{u}), \underline{r} \theta \omega_{\varepsilon}>= \\
p H_{\lambda}(\underline{u})-\lambda \underline{r} q_{1}\left(<\partial D_{1}(\underline{v}), \theta \omega_{\varepsilon}>\right)^{-1}\left(\int_{Q} b(x, t)(\underline{u})^{p-1} \theta \omega_{\varepsilon} d x d t\right) \geq \\
p H_{\lambda}(\underline{u})+\lambda \underline{r} q_{1}\left[\eta\left[\int_{0}^{T} \theta^{h+1} \omega_{\varepsilon} d t\right) \int_{\Omega}(\underline{v})^{q_{1}-1} d x\right]^{-1}\left(\int_{0}^{T} \theta^{2} \omega_{\varepsilon} d t\right) \int_{\Omega}(\underline{u})^{p-1} d x .
\end{gathered}
$$

Let us add

$$
\begin{gathered}
\left(\int_{0}^{T} \theta^{h+1} \omega_{\varepsilon} d t\right)^{-1} \int_{0}^{T} \theta^{2} \omega_{\varepsilon} d t=\left(\theta^{h-1}\left(t_{\varepsilon}\right)\right)^{-1} \text { where } t_{\varepsilon} \in\left[t_{0}-2 \varepsilon, t_{0}+2 \varepsilon\right] \\
{\left[\text { resp. }\left(\int_{0}^{T} \theta^{h+1} \omega_{\varepsilon} d t\right)^{-1} \int_{0}^{T} \theta^{2} \omega_{\varepsilon} d t \geq\left(\max \left\{\theta^{h-1}\left(t_{\varepsilon}^{\prime}\right), \theta^{h-1}\left(t_{\varepsilon}^{\prime \prime}\right)\right\}\right)^{-1} \text { where } t_{\varepsilon}^{\prime} \in[0,2 \varepsilon] \text { and } t_{\varepsilon}^{\prime \prime} \in[T-2 \varepsilon, T]\right] .}
\end{gathered}
$$


Since as $\varepsilon \rightarrow 0^{+}$

$$
\theta^{h-1}\left(t_{\varepsilon}\right) \rightarrow \theta^{h-1}\left(t_{0}\right)=0\left[\operatorname{resp} . \theta^{h-1}\left(t_{\varepsilon}^{\prime}\right) \rightarrow \theta^{h-1}(0)=0 \text { and } \theta^{h-1}\left(t_{\varepsilon}^{\prime \prime}\right) \rightarrow \theta^{h-1}(T)=0\right],
$$

from (3.28) we get that it is possible to choose $\varepsilon$ such that (3.27) holds with $\tilde{v}=\theta \omega_{\varepsilon}$.

Application 3.3 (connected to Theor.2.2 (case $\left(\mathrm{c}_{2}\right)$ ) and Theor.2.3 (case $\left(\mathrm{c}_{9}\right)$ with $\left.\mathrm{m}_{1}=1\right)$ ). We premise some clarifications. Let

$$
\frac{2 N}{N+2} \leq p \leq \frac{2 N}{N-2} \text { if } N>2,1<p<\infty \text { if } N=2
$$

We note

$$
(3.29) \Rightarrow W_{0}^{1}(\Omega) \subseteq L^{p}(\Omega) \cap L^{p^{\prime}}(\Omega) \text { with continuous embedding }\left(p^{\prime}=p /(p-1)\right)
$$

Let $X=W^{2, p}(\Omega) \cap W_{0}^{1}(\Omega)$. We note the norm on $\mathrm{X}$

$$
\|z\|_{X}=\left(\int_{\Omega}|\Delta z|^{p} d x\right)^{\frac{1}{p}}
$$

is equivalent to the natural one on $\mathrm{X}$. It is also equivalent to the norm of $W^{2, p}(\Omega)$ on $X$. In fact, there exist $c_{1}, c_{2}, c_{3}>0$ such that for any $z \in X$

$$
\begin{aligned}
& \|z\|_{W^{2, p}(\Omega)} \leq c_{1}\left(\|\Delta z\|_{L^{p}(\Omega)}+\|z\|_{L^{p}(\Omega)}\right)([2], \text { Theor.8.2 page 444) } \\
& \|z\|_{L^{p}(\Omega)} \leq c_{2}\|\nabla z\|_{L^{2}(\Omega)} \leq c_{3}\|\Delta z\|_{L^{p}(\Omega)}(\text { from }(3.30))
\end{aligned}
$$

Therefore $X$ is a closed subspace of $W^{2, p}(\Omega)$. It is easy to verify that

$$
\exists z^{*} \in X: \int_{\Omega}\left|\nabla z^{*}\right|^{p} d x=1 \text { and } \int_{\Omega}\left|\Delta z^{*}\right|^{p} d x=\inf \left\{\int_{\Omega}|\Delta z|^{p} d x: z \in X \text { and } \int_{\Omega}|\nabla z|^{p} d x=1\right\}
$$

then, set $\lambda^{*}=\int_{\Omega}\left|\Delta z^{*}\right|^{p} d x>0$, we have

$$
\begin{gathered}
\int_{\Omega}|\Delta z|^{p} d x \quad \geq \lambda^{*} \int_{\Omega}|\nabla z|^{p} d x \forall z \in X \\
\left.\int_{\Omega}|\Delta z|^{p} d x \quad-\lambda \int_{\Omega}|\nabla z|^{p} d x \geq\left(1-\lambda\left(\lambda^{*}\right)^{-1}\right) \int_{\Omega}|\Delta z|^{p} d x \quad \forall \lambda \in\right] 0, \lambda^{*}[\text { and } \forall z \in X .
\end{gathered}
$$

Let us assume in the definition (1.1) of $W_{T} \quad p_{1}=p_{2}=p, n=2$ and $\mathrm{V}=\mathrm{X}$, then

$$
\|v\|=\left(\int_{Q}\left|\frac{\partial v}{\partial t}\right|^{p} d x d t+\int_{Q}|\Delta v|^{p} d x d t\right)^{1 / p} \forall v \in W_{T}
$$

and let us set for any $v \in W_{T}$

$$
A(v)=p^{-1}\|v\|^{p}, B(v)=p^{-1} \int_{Q}|\nabla v|^{p} d x d t
$$




$$
D_{1}(v)=q_{1}^{-1} \int_{Q} d_{1}(x, t)|\nabla v|^{q_{1}} d x d t, \quad D_{j}(v)=-q_{j}^{-1} \int_{Q} d_{j}(x, t)|v|^{q_{j}} d x d t \quad \text { as } j=2, \ldots, m
$$

where

$$
\begin{gathered}
1<q_{1}<\ldots<q_{m}<p ; d_{j} \in\left(\mathbb{P}_{T}(\Omega x \mathbb{R}) \cap L^{\infty}(Q)\right) \backslash\{0\} \text { as } j=1, \ldots m, \\
d_{j} \geq 0 \text { a.e. in } Q \text { as } j=2, \ldots m .
\end{gathered}
$$

Problem $\left(P^{T}\right)$ becomes:

Find $u \in W_{T} \backslash\{0\}$ such that

$$
\begin{gathered}
\int_{Q}\left|\frac{\partial u}{\partial t}\right|^{p-2} \frac{\partial u}{\partial t} \frac{\partial v}{\partial t} d x d t+\int_{Q}|\Delta u|^{p-2} \Delta u \Delta v d x d t=\lambda \int_{Q}|\nabla u|^{p-2} \nabla u \nabla v d x d t+\int_{Q} d_{1}(x, t)|\nabla u|^{q_{1}-2} \nabla u \nabla v d x d t- \\
\sum_{j=2}^{m} \int_{Q} d_{j}(x, t)|u|^{q_{j}-2} u v d x d t \quad \forall v \in W_{T} .
\end{gathered}
$$

Each solution u of (3.34) is for definition a weak solution of the problem:

$$
\begin{aligned}
& -\frac{\partial}{\partial t}\left(\left|\frac{\partial u}{\partial t}\right|^{p-2} \frac{\partial u}{\partial t}\right)+\Delta\left(|\Delta u|^{p-2} \Delta u\right)=-\lambda \operatorname{div}\left(|\nabla u|^{p-2} \nabla u\right)-\operatorname{div}\left(d_{1}(x, t)|\nabla u|^{q_{1}-2} \nabla u\right)-\sum_{j=2}^{m} d_{j}(x, t)|u|^{q_{j}-2} u \quad \text { in } Q \\
& u=0 \text { and }|\Delta u|^{p-2} \Delta u=0 \quad \text { on } \Sigma, \\
& u(x, 0)=u(x, T) \text { and } \frac{\partial u}{\partial t}(x, 0)=\frac{\partial u}{\partial t}(x, T) \text { on } \Omega .
\end{aligned}
$$

Let us introduce the conditions

There exist a compact set $\mathrm{K} \subseteq\left[0, T\left[\right.\right.$ with $|\mathrm{K}|_{1}>0$ and an open

$$
\begin{aligned}
& \text { set } \Omega^{+} \subseteq \Omega \text { such that } d_{1}(x, t)>0 \text { a.e.in } \Omega^{+} x \mathrm{~K}, \\
& \int_{0}^{T} d_{1}(x, t) d t<0 \text { a.e. in } \Omega .
\end{aligned}
$$

We note

$$
(3.36) \Rightarrow V^{+}\left(D_{1}\right) \neq \varnothing,(3.37) \Rightarrow \frac{\partial v}{\partial t} \sim 0 \text { in } \mathrm{Q} \forall v \in V^{+}\left(D_{1}\right)
$$

besides

$$
(3.37) \Rightarrow V_{\lambda}^{-} \cap S\left(D_{1}\right) \neq \varnothing \forall \lambda>\lambda^{*},
$$

since as $v=\left|D_{1}\left(z^{*}\right)\right|^{-\frac{1}{q_{1}}} z^{*}$ we have $D_{1}(v)=-1$ and $H_{\lambda}(v)<0$.

Taking into account from (3.32)

$$
p^{-1}\left[\int_{Q}\left|\frac{\partial v}{\partial t}\right|^{p} d x d t+\int_{Q}|\Delta v|^{p} d x d t-\lambda \int_{Q}|\nabla v|^{p} d x d t\right] \geq p^{-1}\left[\int_{Q}\left|\frac{\partial v}{\partial t}\right|^{p} d x d t+\left(1-\lambda\left(\lambda^{*}\right)^{-1}\right) \int_{Q}|\Delta v|^{p} d x d t\right]
$$




$$
\forall \lambda \in] 0, \lambda^{*}\left[\text { and } \forall v \in W_{T}\right.
$$

we have

$$
\left.\left(i_{21}\right) \text {, in particular }\left(i_{22}\right), \text { holds if } \lambda \in\right]-\infty, \lambda^{*}[\text {. }
$$

Proposition 3.9. Under conditions (3.36) and (3.37), there exists $\delta_{1}^{*}>0$ such that

$$
\left(i_{22}\right) \text { holds if } \lambda \in\left[\lambda^{*}, \lambda^{*}+\delta_{1}^{*}\right] \text {. }
$$

Proof. Reasoning by contradiction, for any $k \in \mathrm{N}$ there exist $v_{k} \in V^{+}\left(D_{1}\right)$ and $\lambda_{k} \in\left[\lambda^{*}, \lambda^{*}+k^{-1}\right]$ such that

$$
\left\|v_{k}\right\|^{p}-\lambda_{k} \int_{Q}\left|\nabla v_{k}\right|^{p} d x d t<p k^{-1}\left\|v_{k}\right\|^{p}
$$

Then with $w_{k}=\left\|v_{k}\right\|^{-1} v_{k}$ and $w \in W_{T}$ such that (within a subsequence) $w_{k} \rightarrow$ w weakly in $W_{T} \quad$, from the relations

$$
\int_{Q}\left|\frac{\partial w_{k}}{\partial t}\right|^{p} d x d t+\int_{Q}\left|\Delta w_{k}\right|^{p} d x d t-\lambda_{k} \int_{Q}\left|\nabla w_{k}\right|^{p} d x d t<p k^{-1}, \int_{Q} d_{1}(x, t)\left|\nabla w_{k}\right|^{q_{1}} d x d t>0
$$

passing to limit as $k \rightarrow+\infty$ we get

$$
\begin{aligned}
& \int_{Q}\left|\frac{\partial w}{\partial t}\right|^{p} d x d t+\int_{Q}|\Delta w|^{p} d x d t-\lambda^{*} \int_{Q}|\nabla w|^{p} d x d t \leq 0, \\
& \int_{Q} d_{1}(x, t)|\nabla w|^{q_{1}} d x d t \geq 0 .
\end{aligned}
$$

Since from (3.31) $\int_{Q}|\Delta w|^{p} d x d t \geq \lambda^{*} \int_{Q}|\nabla w|^{p} d x d t$, according to (3.38) we get $\frac{\partial w}{\partial t} \equiv 0$ in Q. Consequently

$$
(3.37) \text { and }(3.39) \Rightarrow|\nabla w| \equiv 0 \text { in } Q, \text { that is } w \equiv 0 \text { in } Q
$$

from which the contradiction $1=\lim _{k \rightarrow+\infty}\left\|w_{k}\right\|^{p} \leq \lim _{k \rightarrow+\infty}\left(\lambda_{k} \int_{Q}\left|\nabla w_{k}\right|^{p} d x d t+p k^{-1}\right)=0$.

Proposition 3.10. Under conditions (3.37), there exists $\delta_{2}^{*}>0$ such that

$$
\left.\left.\left(i_{24}\right) \text { holds with } m_{1}=1 \text { if } \lambda \in\right] \lambda^{*}, \lambda^{*}+\delta_{2}^{*}\right] \text {. }
$$

Proof. Reasoning by contradiction, as in Prop.3.5 there exist $\left(\lambda_{k}\right)_{k \in \mathrm{N}}$, with $\left.\left.\lambda_{k} \in\right] \lambda^{*}, \lambda^{*}+k^{-1}\right]$, and $\left(v_{k, h_{k}}\right)_{k \in \mathrm{N}} \subseteq W_{T}$ such that

$$
H_{\lambda_{k}}\left(v_{k, h_{k}}\right)<0, D_{1}\left(v_{k, h_{k}}\right)=-1, \lim _{k \rightarrow+\infty}\left\|v_{k, h_{k}}\right\|=+\infty .
$$

Then, set $w_{k}=\left\|v_{k, h_{k}}\right\|^{-1} v_{k, h_{k}}$, we have

$$
\int_{Q}\left|\frac{\partial w_{k}}{\partial t}\right|^{p} d x d t+\int_{Q}\left|\Delta w_{k}\right|^{p} d x d t<\lambda_{k} \int_{Q}\left|\nabla w_{k}\right|^{p} d x d t
$$




$$
\int_{Q} d_{1}(x, t)\left|\nabla w_{k}\right|^{q_{1}} d x d t=-q_{1}\left\|v_{k, h_{k}}\right\|^{-q_{1}} \rightarrow 0 \text { as } k \rightarrow+\infty .
$$

Let $w \in W_{T}$ such that (within a subsequence) $w_{k} \rightarrow$ w weakly in $W_{T}$. Relations (3.40), (3.41) imply

$$
\int_{Q}\left|\frac{\partial w}{\partial t}\right|^{p} d x d t+\int_{Q}|\Delta w|^{p} d x d t-\lambda^{*} \int_{Q}|\nabla w|^{p} d x d t \leq 0, \int_{Q} d_{1}(x, t)|\nabla w|^{q_{1}} d x d t=0,
$$

from which, taking into account (3.37), we deduce that $w \equiv 0$ in $\mathrm{Q}$ and the contradiction $1=\lim _{k \rightarrow+\infty}\left\|w_{k}\right\|^{p} \leq \lim _{k \rightarrow+\infty} \lambda_{k} \int_{Q}\left|\nabla w_{k}\right|^{p} d x d t=0$

How established far allows the following result.

Proposition 3.11 (Theor.2.2(case $\left(c_{2}\right)$ ); Theor.(2.3) (case $\left(c_{9}\right)$ with $m_{1}=1$ )). Under assumptions (3.29) and (3.33) we have: when (3.36) and (3.37) hold, with $\left.\lambda \in]-\infty, \lambda^{*}+\delta_{1}^{*}\right]$ problem (3.35) has at least two nonstationary weak solutions $u_{0}$ and $-u_{0}\left(u_{0}=r_{0} v_{0}, r_{0}=\right.$ const. $\left.>0, v_{0} \in S_{\lambda} \cap V^{+}\left(D_{1}\right)\right)$;

when (3.37) holds, with $\left.\lambda \in] \lambda^{*}, \lambda^{*}+\delta_{2}^{*}\right]$ problem (3.35) has at least two weak solutions $\underline{u}$ and $-\underline{u}$ ( $\underline{u}=\underline{r} \underline{v}$, $\underline{r}=$ const. $\left.>0, \underline{v} \in V_{\lambda}^{-} \cap S\left(D_{1}\right)\right)$.

Consequently, when (3.36) and (3.37) hold, with $\left.\lambda \in] \lambda^{*}, \lambda^{*}+\min \left\{\delta_{1}^{*}, \delta_{2}^{*}\right\}\right]$ problem (3.35) has at least four different weak solutions.

Proposition 3.12. Let $\left.\lambda \in] \lambda^{*}, \lambda^{*}+\delta_{2}^{*}\right]$. If there exist a measurable set $I \subseteq[0, T]$ with $|I|_{1}>0$ and a limit point $t_{0}$ of $\mathrm{I}$ such that $\lim _{\substack{t \rightarrow t_{0} \\ t \in \rho}} d_{1}(x, t) \geq 0$ and as $\mathrm{j}=2, \ldots, \mathrm{m} \lim _{\substack{t \rightarrow t_{9} \\ t \in \rho}} d_{j}(x, t)=0$ a.e. in $\Omega$, then $\underline{u}$ is nonstationary.

Proof. In fact, if $\frac{\partial \underline{u}}{\partial t} \equiv 0$ in $Q$, we get the contradiction

$$
\begin{array}{r}
\int_{\Omega} d_{1}(x, t)|\nabla \underline{u}|^{q_{1}} d x-\sum_{j=2}^{m} \int_{\Omega} d_{j}(x, t)|\underline{u}|^{q_{j}} d x=\int_{\Omega}|\Delta \underline{u}|^{p} d x-\lambda \int_{\Omega}|\nabla \underline{u}|^{p} d x(<0) \text { a.e.in }[0, T], \\
\lim _{\substack{t \rightarrow t_{9} \\
t \in I}}\left(\int_{\Omega} d_{1}(x, t)|\nabla \underline{u}|^{q_{1}} d x-\sum_{j=2}^{m} \int_{\Omega} d_{j}(x, t)|\underline{u}|^{q_{j}} d x\right) \geq 0 .
\end{array}
$$

Let us suppose $d_{1}$ has the following structure (according to (3.36) and (3.37)):

$$
\begin{gathered}
d_{1}(x, t)=d_{11}(x) d_{12}(t), d_{11} \in L^{\infty}(\Omega) \text { and } d_{11}>0 \text { a.e.in } \Omega, d_{12} \in \mathbb{P}_{T}(\mathbb{R}) \cap L^{\infty}(\mathbb{R}), \\
d_{12}^{+} \sim 0 \text { in }[0, T], \int_{0}^{T} d_{12} d t<0
\end{gathered}
$$

there exist $\left.t_{0} \in\right] 0, T\left[\right.$ and $\varepsilon_{0}>0$ as in $\left(c^{*}\right)$ such that $d_{12} \in C^{0}\left(\left[t_{0}-\varepsilon_{0}, t_{0}+\varepsilon_{0}\right]\right)$

and $d_{12}(t)<0 \quad \forall t \in\left[t_{0}-\varepsilon_{0}, t_{0}+\varepsilon_{0}\right]$.

In addition let us suppose:

$$
\forall j \in\{2, \ldots, m\} d_{j}(x, \cdot) \in C^{0}\left(\left[t_{0}-\varepsilon_{0}, t_{0}+\varepsilon_{0}\right]\right) \text { and } d_{j}\left(x, t_{0}\right)=0 \text { a.e.in } \Omega .
$$


Proposition 3.13. Let (3.42) and (3.43) hold. Let $\left.\lambda \in] \lambda^{*}, \lambda^{*}+\delta_{2}^{*}\right]$.If $T<\left(d_{12}\left(t_{0}\right)\right)^{-1} \int_{0}^{T} d_{12} d t$, then $\underline{u}$ is nonstationary.

Proof. It is sufficient (Prop.2.2) to prove that

If $\frac{\partial \underline{u}}{\partial t} \equiv 0$ in $Q$, then there exists $\tilde{v} \in W_{T}$ such that $\left\langle\partial D_{1}(\underline{v}), \tilde{v}>\neq 0\right.$ and

$$
\begin{gathered}
\left.\left[\left\langle\partial H_{\lambda}(\underline{u}), \underline{u}\right\rangle+q_{1}\left(\left\langle\partial D_{1}(\underline{v}), \tilde{v}\right\rangle\right)^{-1}<\partial H_{\lambda}(\underline{u}), \underline{r} \tilde{v}\right\rangle\right]- \\
\sum_{j=2}^{m}\left[\left\langle\partial D_{j}(\underline{u}), \underline{u}\right\rangle+q_{1}\left(\left\langle\partial D_{1}(\underline{v}), \tilde{v}>\right)^{-1}<\partial D_{j}(\underline{u}), \underline{r} \tilde{v}\right\rangle\right] \neq 0 .
\end{gathered}
$$

Let $\omega_{\varepsilon}$ be as in $\left(c^{*}\right)$. We note that

$$
<\partial D_{1}(\underline{v}), \omega_{\varepsilon} \underline{v}>=\left(\int_{0}^{T} d_{12} \omega_{\varepsilon} d t\right) \int_{\Omega} d_{11}(x)|\nabla \underline{v}|^{q_{1}} d x=-q_{1}\left(\int_{0}^{T} d_{12} \omega_{\varepsilon} d t\right)\left(\int_{0}^{T} d_{12} d t\right)^{-1}<0 .
$$

Then

$$
q_{1}\left(<\partial D_{1}(\underline{v}), \omega_{\varepsilon} \underline{v}>\right)^{-1}<\partial H_{\lambda}(\underline{u}), \underline{r} \omega_{\varepsilon} \underline{v}>=-\left(\int_{0}^{T} d_{12} \omega_{\varepsilon} d t\right)^{-1}\left(\int_{0}^{T} \omega_{\varepsilon} d t\right)\left(\int_{0}^{T} d_{12} d t\right)\left(\int_{\Omega}|\Delta \underline{u}|^{p} d x-\lambda \int_{\Omega}|\nabla \underline{u}|^{p} d x\right)
$$

and as $\mathrm{j}=2, \ldots, \mathrm{m}$

$$
q_{1}\left(<\partial D_{1}(\underline{v}), \omega_{\varepsilon} \underline{v}>\right)^{-1}<\partial D_{j}(\underline{u}), \underline{r} \omega_{\varepsilon} \underline{v}>=\left(\int_{0}^{T} d_{12} \omega_{\varepsilon} d t\right)^{-1}\left(\int_{0}^{T} d_{12} d t\right) \int_{Q} d_{j}(x, t)|\underline{u}|^{q_{j}} \omega_{\varepsilon}(t) d x d t .
$$

Let us note that

$$
\left(\int_{0}^{T} d_{12} \omega_{\varepsilon} d t\right)^{-1}\left(\int_{0}^{T} \omega_{\varepsilon} d t\right) \rightarrow\left(d_{12}\left(t_{0}\right)\right)^{-1} \text { as } \varepsilon \rightarrow 0^{+}
$$

besides, set $\eta=\min _{\left[t_{0}-\varepsilon_{0}, t_{0}+\varepsilon_{0}\right]}\left|d_{12}\right|$, we get

$$
\begin{gathered}
\left.\left.\left|\left(\int_{0}^{T} d_{12} \omega_{\varepsilon} d t\right)^{-1} \int_{Q} d_{j}(x, t)\right| \underline{u}\right|^{q_{j}} \omega_{\varepsilon}(t) d x d t\left|=\left(\int_{0}^{T}\left|d_{12}\right| \omega_{\varepsilon} d t\right)^{-1} \int_{Q} d_{j}(x, t)\right| \underline{u}\right|^{q_{j}} \omega_{\varepsilon}(t) d x d t \leq \\
\eta^{-1}\left(\int_{0}^{T} \omega_{\varepsilon} d t\right)^{-1} \int_{0}^{T}\left(\int_{\Omega} d_{j}(x, t)|\underline{u}|^{q_{j}} d x\right) \omega_{\varepsilon}(t) d t \rightarrow \eta^{-1} \int_{\Omega} d_{j}\left(x, t_{0}\right) d x=0 \text { as } \varepsilon \rightarrow 0^{+} .
\end{gathered}
$$

Since

$$
\begin{gathered}
<\partial H_{\lambda}(\underline{u}), \underline{u}>-\left(d_{12}\left(t_{0}\right)\right)^{-1} \int_{0}^{T} d_{12} d t\left(\int_{\Omega}|\Delta \underline{u}|^{p} d x-\lambda \int_{\Omega}|\nabla \underline{u}|^{p} d x\right)= \\
\left(T-\left(d_{12}\left(t_{0}\right)\right)^{-1} \int_{0}^{T} d_{12} d t\right)\left(\int_{\Omega}|\Delta \underline{u}|^{p} d x-\lambda \int_{\Omega}|\nabla \underline{u}|^{p} d x\right)>0,
\end{gathered}
$$




$$
-\sum_{j=2}^{m}<\partial D_{j}(\underline{u}), \underline{u}>=\sum_{j=2}^{m} \int_{Q} d_{j}(x, t)|\underline{u}|^{q_{j}} d x d t \geq 0,
$$

with a suitable $\varepsilon, \tilde{v}=\omega_{\varepsilon} \underline{v}$ fulfills (3.44).

Remark 3.2. It is not difficult to set functions $d_{12}$ as in (3.42) such that $T<\left(d_{12}\left(t_{0}\right)\right)^{-1} \int_{0}^{T} d_{12} d t$.

Application 3.4 (connected to Theor.2.2 (case $\left(\mathrm{c}_{3}\right)$ ) and Theor.2.3 (case $\left(\mathrm{c}_{9}\right)$ with $\mathrm{m}_{1}=\mathrm{m}$ )). Let us assume in the definition (1.1) of $W_{T} \quad p_{1}=p_{2}=2, \mathrm{n}=2$ and $V=W^{2}(\Omega)$, then

$$
\|v\|=\left(\int_{Q}\left(\frac{\partial v}{\partial t}\right)^{2} d x d t+\sum_{|\alpha|=2} \int_{Q}\left(D^{\alpha} v\right)^{2} d x d t+\int_{Q} v^{2} d x d t\right)^{1 / 2} \forall v \in W_{T}
$$

and let us set as any $v \in W_{T}$

$$
\begin{gathered}
A(v)=2^{-1}\left[\int_{Q}\left(\frac{\partial v}{\partial t}\right)^{2} d x d t+\sum_{|\alpha|=2} \int_{Q}\left(D^{\alpha} v\right)^{2} d x d t\right] \\
\left.B(v)=2^{-1} \int_{Q} b_{1}(x, t)\left(v^{+}\right)^{2}-b_{2}(x, t)\left(v^{-}\right)^{2}\right) d x d t \quad\left(v^{-}=\min \{v, 0\}\right), \\
D_{j}(v)=-q_{j}^{-1}\left(\int_{Q}|\nabla v|^{\gamma_{j}} d x d t\right)^{q_{j} / \gamma_{j}} \text { as } j=1, \ldots, m-1, \\
D_{m}(v)=(2 n)^{-1}\left(\int_{Q} d_{m}(x, t) v^{2} d x d t\right)^{n},
\end{gathered}
$$

where

$1<\gamma_{j} \leq 2, n$ is a positive odd integer, $2<q_{1}<\ldots<q_{m-1}<q_{m}=2 n$;

$$
\text { as } i=1,2 b_{i} \text { and } d_{m} \in\left(\mathrm{P}_{T}(\Omega x \mathbb{R}) \cap L^{\infty}(Q)\right) \backslash\{0\}, b_{i}(x, t)>0 \text { a.e. in } Q \text {. }
$$

Problem $\left(P^{T}\right)$ becomes:

Find $u \in W_{T} \backslash\{0\}$ such that

$$
\begin{gathered}
\int_{Q} \frac{\partial u}{\partial t} \frac{\partial v}{\partial t} d x d t+\sum_{|\alpha|=2} \int_{Q} D^{\alpha} u D^{\alpha} v d x d t=\lambda \int_{Q}\left(b_{1}(x, t) u^{+} v-b_{2}(x, t) u^{-} v\right) d x d t- \\
\sum_{j=1}^{m-1}\left(\int_{Q}|\nabla u|^{\gamma_{j}} d x d t\right)^{\frac{q_{j}}{\gamma_{j}}-1}\left(\int_{Q}|\nabla u|^{\gamma_{j}-2} \nabla u \nabla v d x d t\right)+\left(\int_{Q} d_{m}(x, t) u^{2} d x d t\right)^{n-1} \int_{Q} d_{m}(x, t) u v d x d t \quad \forall v \in W_{T} .
\end{gathered}
$$

Each solution u of (3.46) is for definition a weak solution of the problem:

$$
-\frac{\partial^{2} u}{\partial t^{2}}+\sum_{|\alpha|=2} D^{\alpha} D^{\alpha} u=\lambda\left(b_{1}(x, t) u^{+}-b_{2}(x, t) u^{-}\right)+\sum_{j=1}^{m-1}\left(\int_{Q}|\nabla u|^{\gamma_{j}} d x d t\right)^{\frac{q_{j}}{\gamma_{j}}-1} \operatorname{div}\left(|\nabla u|^{\gamma_{j}-2} \nabla u\right)+
$$




$$
\begin{gathered}
\left(\int_{Q} d_{m}(x, t) u^{2} d x d t\right)^{n-1} d_{m}(x, t) u \quad \text { in } Q, \\
\frac{\partial \Delta u}{\partial \nu}=\left[\sum_{j=1}^{m-1}\left[\int_{Q}|\nabla u|^{\gamma_{j}} d x d t\right]^{\frac{q_{j}}{\gamma_{j}}-1}|\nabla u|^{\gamma_{j}-2}\right] \frac{\partial u}{\partial \nu} \quad \text { on } \Sigma, \\
\frac{\partial}{\partial \nu}\left(\frac{\partial u}{\partial x_{h}}\right)=0 \quad \text { as } h=1, \ldots, N \text { on } \Sigma, \\
u(x, 0)=u(x, T) \text { and } \frac{\partial u}{\partial t}(x, 0)=\frac{\partial u}{\partial t}(x, T) \text { on } \Omega .
\end{gathered}
$$

Let us introduce the conditions

$$
\begin{gathered}
d_{m}^{+\sim 0} \text { in } \mathrm{Q}, \\
\int_{0}^{T} d_{m}(x, t) d t<0 \text { a.e. in } \Omega .
\end{gathered}
$$

Evidently

$$
(3.48) \Rightarrow V^{+}\left(D_{m}\right) \neq \varnothing,(3.49) \Rightarrow \frac{\partial v}{\partial t} \smile 0 \text { in } \mathrm{Q} \forall v \in V^{+}\left(D_{m}\right),(3.49) \Rightarrow V_{\lambda}^{-} \cap S\left(D_{m}\right) \neq \varnothing \forall \lambda>0 .
$$

Reasoning as in Prop.3.9 and Prop.3.10, we prove that under conditions (3.48) and (3.49), there exists $\delta_{1}^{*}>0$ such that $\left(i_{23}\right)$ holds if $|\lambda| \leq \delta_{1}^{*}$; under condition (3.49), there exists $\delta_{2}^{*}$ such that $\left(i_{24}\right)$ holds with $m_{1}=m$ if $\left.\left.\lambda \in\right] 0, \delta_{2}^{*}\right]$.

Then

Proposition 3.14. (Theor.2.2 (case $\left(\mathrm{c}_{3}\right)$ ), Theor.2.3 (case $\left(\mathrm{c}_{9}\right)$ with $\left.\mathrm{m}_{1}=\mathrm{m}\right)$ ). Under conditions (3.45) we have:

when (3.48) and (3.49) hold, with $|\lambda| \leq \delta_{1}^{*}$ problem (3.47) has at least one nonstationary weak solution $u_{0}\left(u_{0}=r_{0} v_{0}\right.$, $r_{0}=$ const. $>0, v_{0} \in S_{\lambda} \cap V^{+}\left(D_{m}\right)$ );

when (3.49) holds, with $\left.\lambda \in] 0, \delta_{2}^{*}\right]$ problem (3.47) has at least one weak solution $\underline{u}$ ( $\underline{u}=\underline{r} \underline{v}$, $\underline{r}=$ const. $>0, \underline{v} \in V_{\lambda}^{-} \cap S\left(D_{m}\right)$ ).

Consequently, when (3.48) and (3.49) hold, with $\left.\lambda \in] 0, \min \left\{\delta_{1}^{*}, \delta_{2}^{*}\right\}\right]$ problem (3.47) has at least two different weak solutions. Proposition 3.15. Let $\left.\lambda \in] 0, \delta_{2}^{*}\right]$. Let be true one of the following conditions:

There exist a measurable set $I \subseteq[0, T]$ with $|I|_{1}>0$ and a limit point $t_{0}$ of I such that

$$
\lim _{\substack{t \rightarrow t_{0} \\ t \in I^{0}}} d_{m}(x, t)=0 \text { and } \lim _{\substack{t \rightarrow t_{0} \\ t \in I^{0}}} b_{i}(x, t)>0 \text { a.e.in } \Omega \text { as } i=1,2 \text {; }
$$

There exist a measurable set $I \subseteq[0, T]$ with $|I|_{1}>0$, a limit point $t_{0}$ of I and $b_{0}>0$ such that

$$
\lim _{\substack{t \rightarrow t_{0} \\ t \in I^{0}}} d_{m}(x, t)=0 \text { a.e.in } \Omega \quad \text { and } b_{i}(x, t) \geq b_{0} \text { a.e.in } \Omega \times I \text { as } i=1,2 \text {. }
$$

Then $\underline{u}$ is nonstationary. 
Proof. If $\frac{\partial \underline{u}}{\partial t} \equiv 0$ in $Q$, then with $\delta=\left(\int_{Q} d_{m}(x, t)(\underline{u})^{2} d x d t\right)^{n-1}>0$ we have

$$
\delta \int_{\Omega} d_{m}(x, t) \underline{u} d x=-\lambda \int_{\Omega}\left(b_{1}(x, t) \underline{u}^{+}-b_{2}(x, t) \underline{u}^{-}\right) d x \text { a.e. } i n[0, T]
$$

from which the contradictions

$$
\begin{gathered}
0=\lim _{\substack{t \rightarrow t_{0} \\
t \in I^{0}}} \int_{\Omega} d_{m}(x, t) \underline{u} d x=-\lambda \lim _{\substack{t \rightarrow t_{0} \\
t \in I^{0}}} \int_{\Omega}\left(b_{1}(x, t) \underline{u}^{+}-b_{2}(x, t) \underline{u}^{-}\right) d x<0 \text { when }(3.50) \text { holds } \\
0=\lim _{\substack{t \rightarrow t_{0} \\
t \in I^{\circ}}} \delta \int_{\Omega} d_{m}(x, t) \underline{u} d x \leq-\lambda b_{0} \int_{\Omega}\left(\underline{u}^{+}-\underline{u}^{-}\right) d x<0 \text { when }(3.51) \text { holds. }
\end{gathered}
$$

Relations (3.48), (3.49) in particular fulfill when

$$
\begin{gathered}
d_{m}(x, t)=d_{m 1}(x) d_{m 2}(t) \text { with } d_{m 1} \in L^{\infty}(\Omega), d_{m 2} \in \mathbb{P}_{T}(\mathbb{R}) \cap L^{\infty}(\mathbb{R}), \\
d_{m 1}>0 \text { a.e. in } \Omega, d_{m 2}^{+} \sim 0 \text { in }[0, T], \int_{0}^{T} d_{m 2} d t<0 .
\end{gathered}
$$

Proposition 3.16. Let (3.52) holds. Let $\left.\lambda \in] 0, \delta_{2}^{*}\right]$. Then $\underline{u}$ is nonstationary.

Proof. Reasoning by contradiction let $\frac{\partial \underline{u}}{\partial t} \equiv 0$ in $Q$. Since $\underline{u} \sim 0$ in $\Omega$, set in (3.46) $u=\underline{u}$ and $v=1$, we have

$$
\left(\int_{Q} d_{m 1}(x) d_{m 2}(t) \underline{u}^{2} d x d t\right)^{n-1} \int_{Q} d_{m 1}(x) d_{m 2}(t) \underline{u} d x d t=-\lambda \int_{Q}\left(b_{1}(x, t) \underline{u}^{+}-b_{2}(x, t) \underline{u}^{-}\right) d x d t<0 .
$$

Then

$$
\left(\int_{Q} d_{m 1}(x) d_{m 2}(t) \underline{u}^{2} d x d t\right)^{n-1}>0
$$

and moreover

$$
\int_{\Omega} d_{m 1}(x) \underline{u} d x>0
$$

since $\int_{0}^{T} d_{m 2} d t<0$. Condition $d_{m 2}^{+} \sim 0$ in $[0, T]$ implies there exists a compact set $\left.\mathbb{K} \subseteq\right] 0, T\left[\right.$ with $|\mathrm{K}|_{1}>0$ and $d_{m 2}>0$ in $\mathrm{K}$.Let $\left(\varphi_{\varepsilon}\right)_{0<\varepsilon<\varepsilon_{0}} \subseteq \mathrm{P}_{T}(\mathbb{R}) \cap C^{\infty}(\mathbb{R}) \quad$ with $\left.0 \leq \varphi_{\varepsilon} \leq 1, \operatorname{supp}_{[0, T]} \varphi_{\varepsilon} \subseteq\right] 0, T\left[\right.$ and $\quad \varphi_{\varepsilon} \rightarrow \chi$ strongly in $L^{s}(] 0, T[)$ as $\varepsilon \rightarrow 0^{+} \forall s \in[1,+\infty[$, where $\chi$ is the chacteristic function of $\mathrm{K}$.

Since $\lim _{\varepsilon \rightarrow 0^{+}} \int_{0}^{T} d_{m 2} \varphi_{\varepsilon} d t=\int_{\mathrm{K}} d_{m 2} d t>0$, we choose $\varepsilon$ such that $\int_{0}^{T} d_{m 2} \varphi_{\varepsilon} d t>0$. Then taking into account (3.53), (3.54), from (3.46) with $u=\underline{u}$ and $v=\varphi_{\varepsilon}$ we get the contradiction

$$
0<\left(\int_{Q} d_{m 1}(x) d_{m 2}(t) \underline{u}^{2} d x d t\right)^{n-1}\left(\int_{0}^{T} d_{m 2} \varphi_{\varepsilon} d t\right) \int_{\Omega} d_{m 1} \underline{u} d x=-\lambda \int_{Q}\left(b_{1}(x, t) \underline{u}^{+}-b_{2}(x, t) \underline{u}^{-}\right) \varphi_{\varepsilon} d x d t<0 .
$$


Application 3.5 (connected to Theor.2.2 (case $\left(\mathrm{c}_{4}\right)$ with $\left.\mathrm{m}_{1}=\mathrm{m}-1\right)$ ). Let us assume in the definition (1.1) of $W_{T} p_{1}=p_{2}=p$ and $V=W_{0}^{n, p}(\Omega)(n=1,2, \ldots)$, then

$$
\|v\|=\left(\int_{Q}\left|\frac{\partial v}{\partial t}\right|^{p} d x d t+\sum_{|\alpha|=n} \int_{Q}\left|D^{\alpha} v\right|^{p} d x d t\right)^{1 / p} \forall v \in W_{T}
$$

and let us set as any $v \in W_{T}$

$$
\begin{gathered}
A(v)=p^{-1}\left[\int_{Q}\left|\frac{\partial v}{\partial t}\right|^{p} d x d t+\sum_{|\alpha|=n} \int_{Q} a(t)\left|D^{\alpha} v\right|^{p} d x d t\right] \\
B(v)=p^{-1} \int_{Q} a(t) b(x)\left(v^{+}\right)^{p} d x d t \\
D_{j}(v)=q_{j}^{-1} \int_{Q} d_{j}(x, t)|v|^{q_{j}} d x d t \quad a s j=1, \ldots, m-1, \\
D_{m}(v)=-q_{m}^{-1} \int_{Q} d_{m}(x, t)|v|^{q_{m}} d x d t
\end{gathered}
$$

where

$$
\begin{gathered}
1<q_{1}<\ldots<q_{m-1}<q_{m} \leq p ; a \in \mathbb{P}_{T}(\mathbb{R}) \cap C^{0}(\mathbb{R}) \text { with } a(t) \geq a_{0} \forall t \in[0, T]\left(a_{0}=\text { const. }>0 \text { and } \leq 1\right), \\
b \in L^{\infty}(\Omega) \backslash\{0\} \text { with } b \geq 0 \text { a.e. in } \Omega, d_{j} \in\left(\mathbb{P}_{T}(\Omega \times \mathbb{R}) \cap L^{\infty}(Q)\right) \backslash\{0\} \text { with } d_{j} \geq 0 \text { a.e. in } Q \\
\text { as } j=1, \ldots, m \text {. }
\end{gathered}
$$

Problem $\left(P^{T}\right)$ becomes:

Find $u \in W_{T} \backslash\{0\}$ such that

$$
\begin{gathered}
\int_{Q}\left|\frac{\partial u}{\partial t}\right|^{p-2} \frac{\partial u}{\partial t} \frac{\partial v}{\partial t} d x d t+\sum_{|\alpha|=n} \int_{Q} a(t)\left|D^{\alpha} u\right|^{p-2} D^{\alpha} u D^{\alpha} v d x d t=\lambda \int_{Q} a(t) b(x)\left(u^{+}\right)^{p-1} v d x d t+ \\
\sum_{j=1}^{m-1} \int_{Q} d_{j}(x, t)|u|^{q_{j}-2} u v d x d t-\int_{Q} d_{m}(x, t)|u|^{q_{m}-2} u v d x d t \quad \forall v \in W_{T} .
\end{gathered}
$$

Each solution $u$ of (3.56) is for definition a weak solution of the problem:

$$
\begin{gathered}
-\frac{\partial}{\partial t}\left(\left|\frac{\partial u}{\partial t}\right|^{p-2} \frac{\partial u}{\partial t}\right)+\sum_{|\alpha|=n}(-1)^{n} a(t) D^{\alpha}\left(\left|D^{\alpha} u\right|^{p-2} D^{\alpha} u\right)=\lambda a(t) b(x)\left(u^{+}\right)^{p-1}+ \\
\sum_{j=1}^{m-1} d_{j}(x, t)|u|^{q_{j}-2} u-d_{m}(x, t)|u|^{q_{m}-2} u \text { in } Q \\
D^{\alpha} u=0 \text { on } \Sigma \text { as } 0 \leq|\alpha| \leq n-1, \\
u(x, 0)=u(x, T) \text { and } \frac{\partial u}{\partial t}(x, 0)=\frac{\partial u}{\partial t}(x, T) \text { on } \Omega .
\end{gathered}
$$

Evidently $\left(i_{21}\right)$ holds if $\left.\left.\lambda \in\right]-\infty, 0\right]$. Let us add that set $\bar{a}=\|a\|_{C^{0}([0, T])}$, there exists $\delta^{*}>0$ satisfying the condition: 


$$
\left.\forall \lambda \in] 0, \delta^{*}\right] \exists c(\lambda)>0: a_{0}\|v\|^{p}-\lambda \bar{a} \int_{Q} b(x)\left(v^{+}\right)^{p} d x d t \geq c(\lambda)\|v\|^{p} \forall v \in W_{T}
$$

In fact, otherwise, for each $k \in \mathbb{N}$ there exist $v_{k} \in W_{T}$ and $\left.\left.\lambda_{k} \in\right] 0, k^{-1}\right]$ such that

$$
a_{0}\left\|v_{k}\right\|^{p}-\lambda_{k} \bar{a} \int_{Q} b(x)\left(v_{k}^{+}\right)^{p} d x d t<k^{-1}\left\|v_{k}\right\|^{p} .
$$

Then with $w_{k}=\left\|v_{k}\right\|^{-1} v_{k}$ we have

$$
a_{0}<\lambda_{k} \bar{a} \int_{Q} b(x)\left(w_{k}^{+}\right)^{p} d x d t+k^{-1}
$$

from which, passing to limit as $k \rightarrow+\infty$, we get $a_{0} \leq 0$.

(3.58) implies $\left(i_{21}\right)$ holds even if $\left.\left.\lambda \in\right] 0, \delta^{*}\right]$.

Proposition 3.17. (Theor.2.2 (case $\left(\mathrm{c}_{4}\right)$ with $\left.\mathrm{m}_{1}=\mathrm{m}-1\right)$ ). Under conditions (3.55), with $\left.\left.\lambda \in\right]-\infty, \delta^{*}\right]$ problem (3.57) has at least one weak solution $u_{0}\left(u_{0}=r_{0} v_{0}, r_{0}=\right.$ const. $\left.>0, v_{0} \in S_{\lambda} \cap V^{+}\left(D_{1}, \ldots, D_{m-1}\right)\right)$.

About the nonstationarity of $u_{0}$, let us introduce the conditions:

There exist a measurable set $I \subseteq[0, T]$ with $|I|_{1}>0$ and a limit point $t_{0}$ of I such that

$$
\lim _{\substack{t \rightarrow t_{0} \\ t \in I}} d_{j}(x, t)=0 \text { for almost any } x \in \Omega \text { and as } \mathrm{j}=1, \ldots, \mathrm{m}-1
$$

There exist an open interval $I \subseteq[0, T], g \in C^{0}(I)$ with $g(t)>0 \forall t \in I$ and $g \sim \eta a$ in I $\forall \eta>0, g_{j} \in L^{\infty}(\Omega)$ with $g_{j}>0$ a.e. in $\Omega$ such that $d_{j}(x, t)=g_{j}(x) g(t)$ as almost every $x \in \Omega$ and as each

$$
t \in I(j=1, \ldots, m)
$$

There exist $\left.t_{0} \in\right] 0, T\left[\right.$ and $\varepsilon_{0}>0$ as in $\left(c^{*}\right)$ such that for almost any $x \in \Omega \quad d_{j}(x, \cdot) \in C^{0}\left(\left[t_{0}-\varepsilon_{0}, t_{0}+\varepsilon_{0}\right]\right) a s j=1, \ldots, m$ and

$$
\begin{aligned}
& \sum_{j=1}^{m-1} \int_{Q}\left[d_{j}(x, t)-a(t)\left(a\left(t_{0}\right)\right)^{-1} d_{j}\left(x, t_{0}\right)\right]\left|u_{0}\right|^{q_{j}} d x d t- \\
& \qquad \int_{Q}\left[d_{m}(x, t)-a(t)\left(a\left(t_{0}\right)\right)^{-1} d_{m}\left(x, t_{0}\right)\right]\left|u_{0}\right|^{q_{m}} d x d t \neq 0 .
\end{aligned}
$$

Remark 3.3. It is easy to find assumptions on $d_{j}$ and $a$ such that the inequality in (3.61) holds.

Proposition 3.18. Let $\left.\lambda \in]-\infty, \delta^{*}\right]$. If one of the conditions (3.59) - (3.61) holds, then $u_{0}$ is nonstationary.

Proof. Reasoning by contradiction, let $\frac{\partial u_{0}}{\partial t} \equiv 0$ in Q.

When (3.59) holds, we have the contradiction

$$
0=\lim _{\substack{t \rightarrow t_{0} \\ t \in I}} \sum_{j=1}^{m-1} \int_{\Omega} d_{j}(x, t)\left|u_{0}\right|^{q_{j}} d x \geq \delta
$$

where

$$
\delta=a_{0} \sum_{|\alpha|=n} \int_{\Omega}\left|D^{\alpha} u_{0}\right|^{p} d x>0 \text { if } \lambda \leq 0
$$




$$
\left.\left.\delta=a_{0} \sum_{|\alpha|=n} \int_{\Omega}\left|D^{\alpha} u_{0}\right|^{p} d x-\lambda \bar{a} \int_{\Omega} b(x)\left(u_{0}^{+}\right)^{p} d x>0 \quad \text { (from (3.58)) if } \lambda \in\right] 0, \delta^{*}\right] .
$$

When (3.60) holds, we have $g(t)=\eta_{0} a(t) \forall t \in I$ with

$$
\eta_{0}=\left[\sum_{|\alpha|=n} \int_{\Omega}\left|D^{\alpha} u_{0}\right|^{p} d x-\lambda \int_{\Omega} b(x)\left(u_{0}^{+}\right)^{p} d x\right]\left[\left.\left|\sum_{j=1}^{m-1} \int_{\Omega} g_{j}(x)\right| u_{0}\right|^{q_{j}} d x-\int_{\Omega} g_{m}(x)\left|u_{0}\right|^{q_{m}} d x\right]^{-1}>0,
$$

and this contradicts hypothesis.

When (3.61) holds, it is sufficient(Prop.2.1) to prove that

There exists $\tilde{v} \in W_{T}$ such that $\left\langle\partial H_{\lambda}\left(v_{0}\right), \tilde{v}\right\rangle \neq 0$ and

$$
\sum_{j=1}^{m}\left[<\partial D_{j}\left(u_{0}\right), u_{0}>-p\left(<\partial H_{\lambda}\left(v_{0}\right), \tilde{v}>\right)^{-1}<\partial D_{j}\left(u_{0}\right), r_{0} \tilde{v}>\right] \neq 0 .
$$

With $\omega_{\varepsilon}$ as in $\left(c^{*}\right)$ we have

$$
\begin{aligned}
& <\partial H_{\lambda}\left(v_{0}\right), \omega_{\varepsilon} v_{0}>=\sum_{|\alpha|=n} \int_{Q} a(t)\left|D^{\alpha} v_{0}\right|^{p} \omega_{\varepsilon} d x d t-\lambda \int_{Q} a(t) b(x)\left(v_{0}^{+}\right)^{p} \omega_{\varepsilon} d x d t= \\
& \left(\int_{0}^{T} a \omega_{\varepsilon} d t\right)\left[\left.\left|\sum_{|\alpha|=n} \int_{\Omega}\right| D^{\alpha} v_{0}\right|^{p} d x-\lambda \int_{\Omega} b(x)\left(v_{0}^{+}\right)^{p} d x\right]=p\left(\int_{0}^{T} a \omega_{\varepsilon} d t\right)\left(\int_{0}^{T} a d t\right)^{-1}>0 .
\end{aligned}
$$

Then

$$
\begin{gathered}
\sum_{j=1}^{m}\left[<\partial D_{j}\left(u_{0}\right), u_{0}>-p\left(<\partial H_{\lambda}\left(v_{0}\right), \omega_{\varepsilon} v_{0}>\right)^{-1}<\partial D_{j}\left(u_{0}\right), r_{0} \omega_{\varepsilon} v_{0}>\right]= \\
\sum_{j=1}^{m-1}\left[\int_{Q} d_{j}(x, t)\left|u_{0}\right|^{q_{j}} d x d t-\left(\int_{0}^{T} a d t\right)\left(\int_{0}^{T} a \omega_{\varepsilon} d t\right)^{-1} \int_{Q} d_{j}(x, t)\left|u_{0}\right|^{q_{j}} \omega_{\varepsilon}(t) d x d t\right]- \\
{\left[\int_{Q} d_{m}(x, t)\left|u_{0}\right|^{q_{m}} d x d t-\left(\int_{0}^{T} a d t\right)\left(\int_{0}^{T} a \omega_{\varepsilon} d t\right)^{-1} \int_{Q} d_{m}(x, t)\left|u_{0}\right|^{q_{m}} \omega_{\varepsilon}(t) d x d t\right] .}
\end{gathered}
$$

Since as $\mathrm{j}=1, \ldots, \mathrm{m}$

$$
\begin{gathered}
\lim _{\varepsilon \rightarrow 0^{+}}\left(\int_{0}^{T} a d t\right)\left(\mid \int_{0}^{T} a \omega_{\varepsilon} d t\right)^{-1} \int_{Q} d_{j}(x, t)\left|u_{0}\right|^{q_{j}} \omega_{\varepsilon}(t) d x d t= \\
\left(\int_{0}^{T} a d t\right)\left(a\left(t_{0}\right)\right)^{-1} \int_{\Omega} d_{j}\left(x, t_{0}\right)\left|u_{0}\right|^{q_{j}} d x=\int_{Q} a(t)\left(a\left(t_{0}\right)\right)^{-1} d_{j}\left(x, t_{0}\right)\left|u_{0}\right|^{q_{j}} d x d t
\end{gathered}
$$

with a suitable $\varepsilon, \tilde{v}=\omega_{\varepsilon} v_{0}$ fulfills (3.62).

Remark 3.4. It is easy to prove that Propositions 3.17 and 3.18 also hold when

$$
A(v)=p^{-1} \int_{Q}\left[\left|\frac{\partial v}{\partial t}\right|^{\gamma}+(a(t))^{\frac{\gamma}{p}} \sum_{|\alpha|=n}\left|D^{\alpha} v\right|^{\gamma}\right]^{\frac{p}{\gamma}} d x d t \quad \forall v \in W_{T}
$$

with $1<\gamma<p$ and a as in (3.55).

Application 3.6 (connected to Theor.2.2 (case $\left.\left(\mathrm{c}_{6}\right)\right)$ ). Let us assume in the definition (1.1) of $W_{T} \quad p_{1}=p_{2}=p, \mathrm{n}=2$ and $V=W^{2, p}(\Omega)$, then 


$$
\|v\|=\left(\int_{Q}\left|\frac{\partial v}{\partial t}\right|^{p} d x d t+\sum_{|\alpha|=2} \int_{Q}\left|D^{\alpha} v\right|^{p} d x d t+\int_{Q}|v|^{p} d x d t\right)^{1 / p} \forall v \in W_{T},
$$

and let us set as any $v \in W_{T}$

$$
\begin{gathered}
A(v)=p^{-1}\left[\int_{Q}\left|\frac{\partial v}{\partial t}\right|^{p} d x d t+\sum_{|\alpha|=2} \int_{Q} a(t)\left|D^{\alpha} v\right|^{p} d x d t\right], \\
B(v)=p^{-1} \int_{Q} a(t)|v|^{p} d x d t, \\
D_{j}(v)=q_{j}^{-1} \int_{Q} d_{j}(x, t)|\nabla v|^{q_{j}} d x d t \quad \text { as } j=1, \ldots, m-2, \\
D_{m-1}(v)=q_{m-1}^{-1} \int_{Q} d_{m-1}(x, t)|v|^{q_{m-1}} d x d t, \\
D_{m}(v)=q_{m}^{-1} \int_{Q} d_{m}(x, t)|v|^{q_{m}-1} v d x d t,
\end{gathered}
$$

where

$$
\begin{gathered}
1<q_{1}<\ldots<q_{m}<p ; a \in \mathbb{P}_{T}(\mathbb{R}) \cap C^{0}(\mathbb{R}) \text { with } a(t) \geq a_{0} \forall t \in[0, T]\left(a_{0}=\text { const. }>0\right), \\
d_{j} \in\left(\mathbb{P}_{T}(\Omega \times \mathbb{R}) \cap L^{\infty}(Q)\right) \backslash\{0\} \text { as } j=1, \ldots, m, d_{j} \geq 0 \text { a.e. in } Q \text { as } j=1, \ldots, m-2, \\
d_{m-1}>0 \text { and } d_{m}>0 \text { a.e. in } Q \text {. }
\end{gathered}
$$

Problem $\left(P^{T}\right)$ becomes:

Find $u \in W_{T} \backslash\{0\}$ such that

$\int_{Q}\left|\frac{\partial u}{\partial t}\right|^{p-2} \frac{\partial u}{\partial t} \frac{\partial v}{\partial t} d x d t+\sum_{|\alpha|=2} \int_{Q} a(t)\left|D^{\alpha} u\right|^{p-2} D^{\alpha} u D^{\alpha} v d x d t=\lambda \int_{Q} a(t)|u|^{p-2} u v d x d t+\sum_{j=1}^{m-2} \int_{Q} d_{j}(x, t)|\nabla u|^{q_{j}-2} \nabla u \nabla v d x d t+$

$$
\int_{Q} d_{m-1}(x, t)|u|^{q_{m-1}-2} u v d x d t+\int_{Q} d_{m}(x, t)|u|^{q_{m}-1} v d x d t \quad \forall v \in W_{T} .
$$

Each solution u of (3.64) is for definition a weak solution of the problem:

$$
\begin{gathered}
-\frac{\partial}{\partial t}\left(\left|\frac{\partial u}{\partial t}\right|^{p-2} \frac{\partial u}{\partial t}\right)+\sum_{|\alpha|=2} a(t) D^{\alpha}\left(\left|D^{\alpha} u\right|^{p-2} D^{\alpha} u\right)-\lambda a(t)|u|^{p-2} u=-\sum_{j=1}^{m-2} \operatorname{div}\left(d_{j}(x, t)|\nabla u|^{q_{j}-2} \nabla u\right)+ \\
d_{m-1}(x, t)|u|^{q_{m-1}-2} u+d_{m}(x, t)|u|^{q_{m}-1} \text { in } Q, \\
\left.\left.a(t) \sum_{h, k=1}^{N} \frac{\partial}{\partial x_{k}}|| \frac{\partial^{2} u}{\partial x_{h} \partial x_{k}}\right|^{p-2} \frac{\partial^{2} u}{\partial x_{h} \partial x_{k}}\right) \nu_{h}=-\sum_{j=1}^{m-2} d_{j}(x, t)|\nabla u|^{q_{j}-2} \frac{\partial u}{\partial \nu} \text { on } \Sigma, \\
\sum_{k=1}^{N}\left|\frac{\partial^{2} u}{\partial x_{h} \partial x_{k}}\right|^{p-2} \frac{\partial^{2} u}{\partial x_{h} \partial x_{k}} \nu_{k}=0 \text { on } \Sigma \text { as } h=1, \ldots, N,
\end{gathered}
$$




$$
u(x, 0)=u(x, T) \text { and } \frac{\partial u}{\partial t}(x, 0)=\frac{\partial u}{\partial t}(x, T) \text { on } \Omega
$$

We note that

$$
V^{+}\left(D_{1}, \ldots, D_{m-1}\right)=W_{T} \backslash\{0\},\left(i_{21}\right) \text { holds if } \lambda<0
$$

Then

Proposition 3.19. (Theor.2.2 (case ( $\left.\mathrm{c}_{6}\right)$ )).Under conditions (3.63), with $\lambda<0$ problem (3.65) has at least one weak solution $u_{0}\left(u_{0}=r_{0} v_{0}, r_{0}=\right.$ const. $\left.>0, v_{0} \in S_{\lambda}\right)$.

Proposition 3.20. Let $\lambda<0$. Let one of the following conditions be fulfilled:

There exist a measurable set $I \subseteq[0, T]$ with $|I|_{1}>0$ and a limit point $t_{0}$ of I such that

$\lim _{\substack{t \rightarrow t_{\mathrm{t}} \\ t \in 9}} d_{j}(x, t)=0$ for almost any $x \in \Omega$ and as $\mathrm{j}=1, \ldots, \mathrm{m}$;

There exist an open interval $I \subseteq[0, T], g \in C^{0}(I)$ with $g(t)>0 \forall t \in I$ and $g \sim \eta a$ in $\mathrm{I} \forall \eta>0, g_{j} \in L^{\infty}(\Omega)$ with $g_{j}>0$ a.e. in $\Omega$ such that $d_{j}(x, t)=g_{j}(x) g(t)$ as almost every $x \in \Omega$ and as each $t \in I(j=1, \ldots, m)$;

There exist $\left.t_{0} \in\right] 0, T\left[\right.$ and $\varepsilon_{0}>0$ as in $\left(c^{*}\right)$ such that for almost any $x \in \Omega d_{j}(x, \cdot) \in C^{0}\left(\left[t_{0}-\varepsilon_{0}, t_{0}+\varepsilon_{0}\right]\right)$ as $j=.1, \ldots, m-1$ and $\sum_{j=1}^{m-2} \int_{Q}\left[d_{j}(x, t)-a(t)\left(a\left(t_{0}\right)\right)^{-1} d_{j}\left(x, t_{0}\right)\right]\left|\nabla u_{0}\right|^{q_{j}} d x d t+\left.\int_{Q}\left[d_{m-1}(x, t)-a(t)\left(a\left(t_{0}\right)\right)^{-1} d_{m-1}\left(x, t_{0}\right)\right]\left|u_{0}\right|\right|^{q_{m-1}} d x d t \neq 0$, $d_{m}(x, t)=g_{m}(x) a(t)$ as almost each $x \in \Omega$ and for each $t \in[0, T]$ where $g_{m} \in L^{\infty}(\Omega)$ and $g_{m}>0$ a.e.in $\Omega$.

Then $u_{0}$ is nonstationary.

Proof. We reason as in Prop. (3.18).

\section{References}

[1] R.A. Adams, Sobolev spaces, Academic Press (1975).

[2] S.Agmon, The $\mathrm{L}_{\mathrm{p}}$ approach to the Dirichlet problem.I.Ann.Sc.Norm.Sup.Pisa (1959), 405-448.

[3] A.Anane, Simplicité et isolation de la première valeur propre du p-laplacian avec poids, C.R.Acad.Sci,Paris, Sér.I, 305 (1987), 725-728.

[4] C.Q.Dai, Y.Y.Wang, Notes on the equivalence of different variable separation approaches for nonlinear evolution equations, Communications in Nonlinear Science and Numerical Simulations, vol.19 (1) (2014),pp.19-28.

[5] J.Diblik, B.Iričanin, S.Stević, Note on the exixtence of periodic solutions of systems of differential-difference equations, Applied Mathematics. and Computation,vol 232(2014), pp. 922-928

[6] J.L.Lions, Quelques méthodes de résolution des problèmes aux limites non linéaires, Dunod, Paris (1969).

[7] X.Liu, Y.Zhang, H.Shi, Existence of periodic solutions for a class of nonlinear difference equations, Qualitative theory of Dynamical Systems, vol 14,issue 1 (2015), pp.51-69.

[8] Z.Luo, Multiple positive periodic solutions for functional differential equations with impulses and a parameter, Abstract and Applied Analysis, vol 2014, http://dx.doi.org/10.1155/2014/812867.

[9] R.Ma, R.Chen, Z.He, Positive periodic solutions of second-order differential equations with weak singularities, Applied Mathematics. and Computation, vol 232(2014), pp.97-103.

[10] L.Toscano, S.Toscano, On the solvability of a class of general systems of variational equations with nonmonotone operators, Journal of Interdisciplinary Mathematics 14, n.2, (2011), 123-147.

[11] L.Toscano, S.Toscano, Dirichlet and Neumann problems related to nonlinear elliptic systems: solvability, multiple solutions, solutions with positive components, Abstract and Applied Analysis (2012), 1-44.

[12] L.Toscano, S.Toscano, On the solvability of a class of general systems of variational equations with nonmonotone operators: a new result. Applications to Dirichlet and Neumann nonlinear problems, preprint (2015).

[13] S.I. Pohozaev, On periodic solutions to certain nonlinear hyperbolic equations, Dokl. Akad. Nauk SSSR.(1971), vol.198.No 6, pp.1274-1277.

[14] S.I. Pohozaev, On the global fibering method in nonlinear variational problems, Proc.Steklov Inst.of Math, 219 (1997), 281-328.

[15] S.I. Pohozaev, The existence and nonexistence of periodic solutions to certain nonlinear hyperbolic equations, Proceedings of the Steklov Institute of Mathematics, vol.227 (1999), pp.254-279.

[16] E.Zeidler, Nonlinear functional analysis and its applications, II/A, Springer-Verlag (1980). 